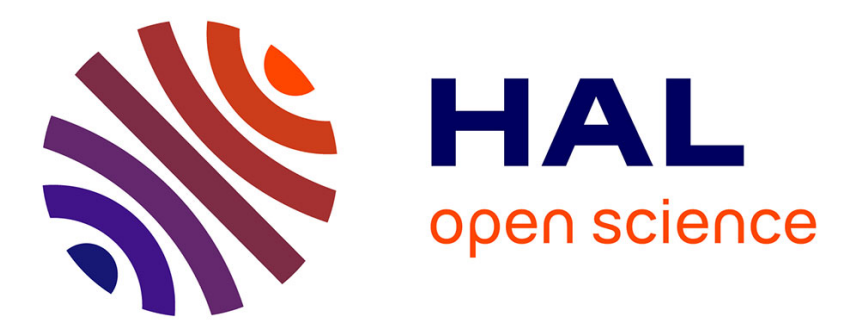

\title{
Non-Minimum Phase Switched Systems: HOSM-Based Fault Detection and Fault Identification via Volterra Integral
}

Hector Rios, Denis Efimov, Jorge Davila, Tarek Raissi, Leonid Fridman, Ali Zolghadri

\section{To cite this version:}

Hector Rios, Denis Efimov, Jorge Davila, Tarek Raissi, Leonid Fridman, et al.. Non-Minimum Phase Switched Systems: HOSM-Based Fault Detection and Fault Identification via Volterra Integral. International Journal of Adaptive Control and Signal Processing, 2013, 10.1002/acs.2448 . hal-00986405

\section{HAL Id: hal-00986405 https://hal.inria.fr/hal-00986405}

Submitted on 5 May 2014

HAL is a multi-disciplinary open access archive for the deposit and dissemination of scientific research documents, whether they are published or not. The documents may come from teaching and research institutions in France or abroad, or from public or private research centers.
L'archive ouverte pluridisciplinaire HAL, est destinée au dépôt et à la diffusion de documents scientifiques de niveau recherche, publiés ou non, émanant des établissements d'enseignement et de recherche français ou étrangers, des laboratoires publics ou privés. 


\title{
Non-Minimum Phase Switched Systems: HOSM-Based Fault Detection and Fault Identification via Volterra Integral Equation*†
}

\author{
H. Ríos ${ }^{1 *}$, D. Efimov ${ }^{2}$, J. Davila ${ }^{3}$, T. Raïssi ${ }^{4}$, L. Fridman ${ }^{1}$ and A. Zolghadri ${ }^{5}$ \\ ${ }^{1}$ National Autonomous University of Mexico, Department of Control Engineering and Robotics, Division of Electrical \\ Engineering, Engineering Faculty, C.P. 04510, México, D.F. Mexico. \\ ${ }^{2}$ The Non-A project at INRIA - LNE, Parc Scientifique de la Haute Borne, 40 avenue Halley, Bt.A Park Plaza 59650 \\ Villeneuve d'Ascq, France. \\ ${ }^{3}$ National Polytechnic Institute, Section of Graduate Studies and Research, ESIME-UPT, C.P. 07340, Mexico D.F. \\ Mexico. \\ ${ }^{4}$ Conservatoire National des Arts et Métiers (CNAM), Cedric-Lab, 292 rue St-Martin, 75141 Paris, France. \\ ${ }^{5}$ University of Bordeaux, IMS-Lab, Automatic Control Group, 351 cours de la libération, 33405 Talence, France.
}

\section{SUMMARY}

In this paper the problem of continuous and discrete state estimation for a class of linear switched systems with additive faults is studied. The class of systems under study can contain non-minimum phase zeroes in some of their "operating modes". The conditions for exact reconstruction of the discrete state are given using structural properties of the switched system. The state-space is decomposed into the strongly observable part, the nonstrongly observable part and the unobservable part, to analyze the effect of the unknown inputs. State observers based on high-order sliding-mode to exactly estimate the strongly observable part and Luenbergerlike observers to estimate the remaining parts are proposed. For the case when the exact estimation of the state cannot be achieved, the ultimate bounds on the estimation errors are provided. The proposed strategy includes a high-order sliding-mode-based fault detection and a fault identification scheme via solution of a Volterra integral equation. The feasibility of the proposed method is illustrated by simulations. Copyright (c) 2013 John Wiley \& Sons, Ltd.

Received ...

KEY WORDS: Switched systems; Fault detection and identification; Non-minimum phase; High-order sliding-mode.

\section{INTRODUCTION}

\subsection{Nomenclature}

The following nomenclature is used in the paper.

- $\mathrm{FD}=$ Fault Detection (FD indicates that something is wrong in the system)

- $\mathrm{FI}=$ Fault Isolation (FI determines the location and the fault type)

- FId = Fault Identification (FId determines the magnitude and shape of the fault)

- FDI $=$ Fault Detection and Isolation

- FDId = Fault Detection and Identification

${ }^{*}$ Correspondence to: H. Ríos, National Autonomous University of Mexico, Department of Control Engineering and Robotics, Division of Electrical Engineering, Engineering Faculty, C.P. 04510, México, D.F., Mexico. Email: hectorrios@comunidad.unam.mx

${ }^{*}$ A preliminary version of this work has been presented in [1].

${ }^{\dagger}$ The work has been done during a research stay of the first author at University of Bordeaux, France. 
- $\mathrm{FTC}=$ Fault Tolerant Control

- HOSM = High-Order Sliding-Mode

- $\mathrm{HS}=$ Hybrid Systems

- LMI = Linear Matrix Inequality

- LTI = Linear Time Invariant

- LSS = Linear Switched Systems

- NSS = Nonlinear Switched Systems

- $\mathrm{NMP}=$ Non-Minimum Phase

- $\mathrm{SMC}=$ Sliding-Mode Control

- $\mathrm{SS}=$ Switched Systems

- VIE $=$ Volterra Integral Equation

\subsection{Antecedents and Motivation}

The HS whose behavior can be represented by the interaction of continuous and discrete dynamics, have been widely studied during the last decades. This kind of systems can be used to describe a wide range of physical and engineering systems. Much attention has been focused on the problems of stability and stabilization (see, for example [2], [3], [4]). An important class of HS is comprised of SS whose dynamics consists of a finite number of subsystems and a logical rule that drives the switchings between these subsystems.

Observation of SS (i.e. the estimation of the continuous and discrete states) is also of great interest in many control areas. This problem has been studied by many authors using different approaches. The main difference is related to the knowledge of the active discrete state: some approaches consider only continuous state uncertainty with known discrete state, while others assume that both of them are unknown. In [5] and [6] a Luenberger observer approach and a HOSM observer for LSS are proposed for the known discrete state case. In [7] the problem of the simultaneous state and input estimation for SS subject to input disturbances is addressed by an algorithm based on the moving horizon estimation method. For unknown discrete state, based on strong detectability and using an LMI approach, in [8] two state observers are designed for some classes of LSS with unknown inputs. To Markovian jump singular systems, another class of SS, in [9] an integral sliding mode observer is designed to estimate the system states, and a sliding mode control scheme is synthesized for the reaching motion based on the state estimates. Considering that the output and an initial state are available, in [10] necessary and sufficient conditions for a SS to be invertible are proposed, i.e. condition for recovering the switching signal and the input uniquely. In the same context, a nonlinear finite time observer to estimate the capacitor voltage for multicellular converters, which have a switched behavior, is proposed by [11]. In [12] a hybrid adaptable observer is proposed that is able to estimate the state for locally Lipschitz systems with application on mechanical oscillators. In [13], based on the nonhomogeneous HOSM approach, a robust observer for the unknown and exogenous switching signal is proposed to solve the problem of continuous and discrete state estimation for a class of NSS. A Nonlinear observer synthesis based on second-order SMC for autonomous SS with jumps is proposed in [14]. In [15] and [16] observability conditions for some classes of SS and a design of hybrid observers to reconstruct both continuous and discrete states are presented. Considering that the continuous state is known, an algorithm for the discrete state reconstruction in uncertain NSS is presented in [17] based on sliding-mode control theory. Sliding mode observers producing suitable residual signals for the problem of simultaneous discrete and continuous state reconstruction in LSS are proposed by [18]. A robust observer is proposed in [19] that is able to estimate the continuous, discrete state, and unknown inputs for autonomous NSS based on HOSM observers.

The issue of model-based FDI in dynamic systems has been an active research area during the last three decades (see, for example [20] and [21]). A typical failure detection process includes residual generation and evaluation. A huge number of publications about FDI problem exist (see for example [22], [23], [24], and the references there in). Residual generation schemes, where the output error between the system and the observer is analyzed to form the residuals, have been extensively studied (see, for example [25], [26] and [27]). In the last decades, observer-based FDI schemes that 
incorporate different control approaches have been developed [28]. In SMC theory the most recent contributions have been presented by [29], [30], [31], [32] and [33]. Most of these works formulate actuator or sensor FId as additional unknown input and exploit the inherent robustness properties of SMC to a certain class of uncertainties, including its ability to directly handle actuator faults.

An FDI methodology that uses structured parity residuals for SS is proposed in [34]. For the feasibility of proposed methodology the authors propose the definition of discernability that is similar to the concept of observability in LTI systems. In [35] based on a hybrid automata model that parameterizes abrupt and incipient faults, an approach for diagnoser design is presented. The diagnostic system architecture that integrates the modeling, prediction, and diagnosis components is described. A methodology for detection and isolation of faults to control SS using a diagnoser is presented in [36]. The notion and conditions of diagnosability of SS in the hybrid input output automata framework is addressed, based on the previous diagnosability notion. The work [37] developed techniques for fault diagnosis in SS based on a knowledge-based and bond graphs approach. In [38] a robust hybrid observer is proposed for a class of uncertain nonlinear SS with unknown mode transition functions, model uncertainties and unknown disturbances. The transition detectability and mode identifiability conditions are studied. Based on hybrid observer, a robust fault diagnosis scheme is presented for faults modeled as discrete modes with unknown transition functions. In [39] a model-based FDI using multiple hypothesis testing is proposed for a stochastic linear SS. A residual generation filter is proposed that generates a residual vector with zero mean and a known covariance when the stochastic linear SS matches the system dynamics. For a class of nonlinear SS with faults and parametric uncertainties, in [40] an observer is designed whose estimation error is not affected by faults, and an observer-based fault tolerant tracking controller is proposed to make the outputs asymptotically track the reference signals for bounded states. In [41] the failure detection and reconstruction is formulated as an unknown input problem and an algorithm for the invertibility of NSS is proposed. A robust hybrid observer for LSS with known active mode, unknown inputs and modeling error is presented in [42]. The proposed observer is synthesized for the task of robust fault detection as a $H_{\infty}$ model-matching problem. Recently, in [43] a residual generator-based robust fault detection filter is used for the problem of design a fault detection system for SS with unknown inputs and known switching signal. The residual generator design is formulated as an optimization problem, and solved iteratively by LMI. In [44] algorithms for robust estimation and FDI for a class of stochastic SS is presented. The robust hybrid estimation algorithm is designed for two kinds of discrete state transition models: the Markov-jump transition model whose discrete transition probabilities are constant and the state-dependent transition model whose discrete state transitions are determined by some guard conditions.

\subsection{Main Contributions}

In the most of the above mentioned schemes, faults are modelled as discrete state, i.e. an SS as the result of the interaction between a system and possible faults (system + faults $=$ SS), and not an SS interacting with faults ( $\mathrm{SS}+$ faults) that is more complicated.

In this paper a solution of the problem of state estimation for LSS subject to additive faults and possibly unstable invariant zeroes (NMP systems with respect to unknown inputs or additive faults) ${ }^{\dagger}$ is presented. State observers based on HOSM to exactly estimate the strongly observable part and Luenberger-like observers to estimate the remaining parts are proposed. The exact estimation of the continuous state allows to realize a finite time and exact estimation of the discrete state in the presence of additive faults. Moreover, the proposed strategy includes a HOSM-based FD composed by a residual generator helped by a bank of observers, and an FId scheme via numerical solution of a VIE.

\footnotetext{
${ }^{\dagger}$ See, for example [45] for the observation problem for a class of NMP causal nonlinear systems.
} 


\subsection{Structure of the paper}

The paper has the following structure. Section 2 deals with the problem statement and some preliminaries are recalled. In section 3 the system transformation is proposed. The observer design is presented through the section 4 . The problem of discrete state estimation is analyzed in section 5. The FDId problem is carried out in section 6. The simulation results are shown in section 7. Finally, some concluding remarks are given in section 8 .

\section{PROBLEM STATEMENT}

Consider the following LSS with faults:

$$
\begin{aligned}
& \dot{x}(t)=A_{j(x(t))} x(t)+B_{j(x(t))} u(t)+E_{j(x(t))} f(t), \\
& y(t)=C_{j(x(t))} x(t),
\end{aligned}
$$

where $x \in \mathcal{X} \subseteq \Re^{n}$ is the state vector, $u \in \mathcal{U} \subseteq \Re^{p}$ is the known input vector, $y \in \mathcal{Y} \subseteq \Re^{m}$ is the output, and $f \in \mathcal{F} \subseteq \Re^{m}$ is the fault vector, which is bounded, i.e. $\|f(t)\| \leq f^{+}<\infty$. The so-called "discrete state" $j(x(t)): \Re \rightarrow \mathcal{Q}=\{1, \ldots, q\}$ determines the current system dynamics among the possible $q$ "operating modes", i.e. $\left\{A_{1}, B_{1}, C_{1}, E_{1}\right\},\left\{A_{2}, B_{2}, C_{2}, E_{2}\right\}, \ldots,\left\{A_{q}, B_{q}, C_{q}, E_{q}\right\}$. The discrete state is generated by a scalar function of the system states (the switching signal) defined as

$$
j(x(t))=\left\{\begin{array}{lll}
1, & \forall x \mid & H x \in \mathcal{H}_{1}, \\
2, & \forall x \mid & H x \in \mathcal{H}_{2}, \\
\vdots & & \\
q, & \forall x \mid & H x \in \mathcal{H}_{q},
\end{array}\right.
$$

where $H$ is a known matrix, $\mathcal{H}_{1}, \mathcal{H}_{2}, \ldots, \mathcal{H}_{q} \subseteq \mathcal{H} \in \Re$ are known convex disjoint subsets, respectively. Notice that for every value of the continuous state $x(t)$ there is only one single value of the discrete state $j(x(t))$, i.e. the discrete state is distinguishable by definition.

In this paper the studied problems are the following:

- Estimation of the continuous state $x(t)$.

- Estimation of the discrete state $j(x(t))$.

- Fault detection.

- Fault identification.

\subsection{Preliminaries}

2.1.1. Notation. The following notation is used. The pseudoinverse matrix of $F \in \Re^{n \times m}$ is defined as $F^{+}=\left(F^{T} F\right)^{-1} F^{T} \in \Re^{m \times n}$. For a matrix $J \in \Re^{n \times m}$ with $n \geq m$ and $\operatorname{rank}(J)=r, J^{\perp} \in$ $\Re^{(n-r) \times n}$ is defined as a matrix such that $\operatorname{rank}\left(J^{\perp}\right)=n-r$ and $J^{\perp} J=0$. The matrix $J^{\perp \perp} \in \Re^{r \times n}$ corresponds to one of the full row rank matrices such that $\operatorname{rank}\left(J^{\perp \perp}\right)=r$ and $J^{\perp}\left(J^{\perp \perp}\right)^{T}=0$. It is clear that the matrices $J^{\perp}$ and $J^{\perp \perp}$ are not unique and that rank $\left[\left(J^{\perp}\right)^{T} \quad\left(J^{\perp \perp}\right)^{T}\right]^{T}=n$. Denote by $\mathcal{L}_{\infty}$ the set of all inputs $\nu$ that satisfy $\|\nu\|<\infty$. Finally, $\lceil\nu\rfloor^{r}=|\nu|^{r} \operatorname{sign}(\nu)$.

2.1.2. Definitions. Some basic definitions for strong observability, strong detectability, invariant zeroes, relative degree and dwell time are introduced in this section (see, for example [46], [47], [48] and [2]).

Consider an LTI system

$$
\begin{aligned}
\dot{x}(t) & =A x(t)+B u(t)+E f(t), \\
y & =C x(t),
\end{aligned}
$$

where $x \in \Re^{n}$ is the state, $y \in \Re^{m}$ is the output, $f \in \Re^{m}$ is an "unknown input" or a fault, and the known matrices $A, C$ and $E$ have corresponding dimensions. In this case, it can be assumed, without loss of generality, that the known input $u(t)$ is equal to zero. 
Definition 1: The system (3) is called strongly observable if for any initial condition $x(0)$ and for all unknown inputs $f(t)$, the identity $y(t) \equiv 0 \forall t \geq 0$ implies that also $x(t) \equiv 0 \forall t \geq 0$.

Definition 2: The system (3) is called strongly detectable if for any initial condition $x(0)$ and for all unknown inputs $f(t)$, the identity $y(t) \equiv 0 \forall t \geq 0$ implies that $x(t) \rightarrow 0$ as $t \rightarrow \infty$.

Definition 3: The complex number $s_{0} \in \mathcal{C}$ is called an invariant zero of the triple $(A, E, C)$ if $\operatorname{rank}\left(R\left(s_{0}\right)\right)<n+\operatorname{rank}(E)$, where $R(s)$ is the Rosenbrock matrix of system (1), i.e.

$$
R(s)=\left[\begin{array}{cc}
s I-A & -E \\
C & 0
\end{array}\right] .
$$

Fact 1: The following statements are equivalent:

i) The system (3) is strongly observable.

ii) The triple $(A, E, C)$ has no invariant zeroes.

Fact 2: The following statements are equivalent:

i) The system (3) is strongly detectable.

ii) The system is minimum-phase (i.e. the invariant zeroes of the triple $(A, E, C)$ satisfy $\operatorname{Re}\{s\}<$ $0)$.

Now, basing on the given statements, we can define the set of unstable invariant zeroes of the system (3) as the set of invariant zeroes of the triple $(A, E, C)$ satisfy $\operatorname{Re}\{s\} \geq 0$. Moreover, notice that if there exist unstable invariant zeroes, then the system (3) is not strongly detectable and nonminimum phase either.

In the case when $E=0$, the notions of strong observability and strong detectability coincide with observability and detectability, respectively. Now, introduce the observability matrix

$$
\mathrm{O}=\left[\begin{array}{llll}
C^{T}, & (C A)^{T}, & \cdots, & \left(C A^{n-1}\right)^{T}
\end{array}\right]^{T} .
$$

Notice that system (3) is observable, independently of the unknown inputs, if and only if $\operatorname{rank}(\mathrm{O})=$ $n$. The unobservable subspace of the pair $(C, A)$ is denoted by $\mathcal{N}$ and it is defined as $\mathcal{N}=\operatorname{ker}(\mathrm{O})$.

Definition 4: The scalar value $\lambda_{0} \in \Im$ is said to be an $(C, A)$-unobservable eigenvalue if

$$
\operatorname{rank}\left(\begin{array}{c}
\lambda_{0} I-A \\
C
\end{array}\right)<n .
$$

Definition 5: A point $x_{0}$ is called weakly unobservable if there exists an input $w(t)$, such that the corresponding output satisfies $y_{w}\left(t, x_{0}\right) \equiv 0 \forall t \geq 0$. The set of all weakly unobservable points of (3) is denoted by $\mathcal{V}^{*}$ and is called the weakly unobservable subspace of (3).

The weakly unobservable subspace satisfies the following relations:

$$
A \mathcal{V}^{*} \subset \mathcal{V}^{*} \oplus \mathcal{E}, \quad C \mathcal{V}^{*}=0
$$

where $\mathcal{E}$ denotes the image of $E$. For any null-output $(A, E)$ - invariant subspace, there exist a map $\bar{K}: \mathcal{X} \rightarrow \mathcal{W}$ such that

$$
(A+E \bar{K}) \mathcal{V}^{*} \subset \mathcal{V}^{*}, \quad C \mathcal{V}^{*}=0 .
$$

Definition 6: The output $y(t)$ is said to have a relative degree vector $\left(r_{1}, \ldots, r_{m}\right)$ with respect to the unknown input $w(t)$ if

$$
\begin{gathered}
c_{i} A^{k} E=0, \forall k<r_{i}-1, \\
c_{i} A^{r_{i}-1} E \neq 0, \forall i=1, \ldots, m .
\end{gathered}
$$


and

$$
\begin{aligned}
& \operatorname{det} Q \neq 0, \\
& Q=\left[\begin{array}{ccc}
c_{1} A^{r_{1}-1} E_{1} & \cdots & c_{1} A^{r_{1}-1} E_{m} \\
& \ddots & \\
c_{m} A^{r_{m}-1} E_{1} & \cdots & c_{m} A^{r_{m}-1} E_{m}
\end{array}\right],
\end{aligned}
$$

where $c_{i}$ is the $i$ - th row of matrix $C$, and $E_{j}$ is the $j$ - th column of matrix $E$.

Definition 7: [2]. The minimal dwell time is a number $T_{\delta}>0$ such that the class of admissible switching signals satisfy the property that the switching times $t_{1}, t_{2}, \ldots$ fulfill the inequality $t_{j+1}-t_{j} \geq T_{\delta}$ for all $j$.

In this paper we study the systems whose hybrid time trajectories satisfies the minimal dwell time definition. Moreover, it is assumed that the dwell time is sufficiently large or it is possible to estimate it (see, for example [49] for the algebraic estimation of the switching times for LSS).

\section{SYSTEM TRANSFORMATION}

Based on the previous definitions, the following assumption ensures the possibility for state estimation:

Assumption 1: All the $\left(A_{j(x(t))}, C_{j(x(t))}\right)$-unobservable eigenvalues satisfy $\operatorname{Re}\{\lambda\}<0$, for all $j \in \mathcal{Q}$ and $t \geq 0$.

It is clear that, as a consequence of the Assumption 1, each subsystem is detectable. Even more, the satisfaction of the above mentioned assumption ensures that the set of unstable invariant zeroes does not belong to the set of $\left(A_{j(x(t))}, C_{j(x(t))}\right)$-unobservable eigenvalues, for all $j \in \mathcal{Q}$.

Now, a suitable transformation to decompose the system into the strongly observable part, the nonstrongly observable part and the unobservable part is applied to each operating mode of the system (1) (see, for example [50] and [48]). For simplicity, there will be omitted the index of the discrete state. However, the transformation is applied for each dynamics generated by the discrete state.

Firstly, let us calculate a basis of the weakly unobservable subspace $\mathcal{V}^{*}$ by means of the computation of the matrices $M_{i}$ defined by the following recursive algorithm ${ }^{\ddagger}$ :

$$
\begin{gathered}
M_{i+1}=N_{i+1}^{\perp \perp} N_{i+1}, \quad M_{1}=C, \\
N_{i+1}=G_{i}\left(\begin{array}{c}
C \\
M_{i} A
\end{array}\right), \quad G_{i}=\left(\begin{array}{c}
0_{p \times q} \\
M_{i} E
\end{array}\right)^{\perp} .
\end{gathered}
$$

The algorithm ends when $\operatorname{rank}\left(M_{i+1}\right)=\operatorname{rank}\left(M_{i}\right)$. Therefore, it is possible to define $M_{n}=$ $M_{n-1}=\ldots=M_{i}$. It was proven in [50] that $\mathcal{V}^{*}=\operatorname{ker}\left(M_{n}\right)$. Now, define $n_{\mathcal{V}}:=\operatorname{rank}\left(M_{n}\right)$ with $M_{n} \in \Re^{n_{\mathcal{V}} \times n}$. Then, form the matrix $V \in \Re^{n \times\left(n-n_{\mathcal{V}}\right)}$ whose columns form a basis of $\mathcal{V}^{*}$.

Secondly, assume that the following assumption is satisfied:

Assumption 2: The output of the system (1) has a relative degree vector $\left(r_{1}, \ldots, r_{m}\right)$ such that $r_{1}+\cdots+r_{m}=n_{\mathcal{V}}$.

Remark 1: Assumption 2 is satisfied for all system, with full relative degree [51]. If it is not satisfied, it is possible to apply alternative methods such that Molinari's algorithm [48].

${ }_{\ddagger}^{\ddagger}$ The matrix $N_{i+1}^{\perp \perp}$ is introduced to excluded the linearly dependent terms of $N_{i+1}$. Therefore, $M_{i+1}$ has full row rank (see [48]). 
According to Definition 6 and Assumption 2, it is possible to form the following matrix $U \in$ $\Re^{n_{\mathcal{V}} \times n}$

$$
\begin{array}{rrrrr}
U=\left[\begin{array}{lllll}
c_{1}^{T}, & \left(c_{1} A\right)^{T}, & \cdots, & \left(c_{1} A^{r_{1}-1}\right)^{T}, & \cdots \\
& c_{m}^{T}, & \left(c_{m} A\right)^{T}, & \cdots, & \left(c_{m} A^{r_{m}-1}\right)^{T}
\end{array}\right]^{T} .
\end{array}
$$

It is easy to see that $\operatorname{rank}(U)=n_{\mathcal{V}}$. Now, from the matrix $U$, form the following matrices $U_{1} \in$ $\Re^{n \mathcal{V}-m \times n}$ and $U_{2} \in \Re^{m \times n}$

$$
\begin{gathered}
U_{1}=\left[\begin{array}{lllll}
c_{1}^{T}, & \left(c_{1} A\right)^{T}, & \cdots, & \left(c_{1} A^{r_{1}-2}\right)^{T}, & \cdots, \\
& c_{m}^{T}, & \left(c_{m} A\right)^{T}, & \cdots & \left(c_{m} A^{r_{m}-2}\right)^{T}
\end{array}\right]^{T}, \\
U_{2}=\left[\begin{array}{llll}
\left(c_{1} A^{r_{1}-1}\right)^{T}, & \cdots, & \left(c_{m} A^{r_{m}-1}\right)^{T}
\end{array}\right]^{T} .
\end{gathered}
$$

Finally, form the matrix $N$ whose columns form a basis of the unobservable subspace $\mathcal{N}$. It is clear by Definition 5 that $\mathcal{N} \subset \mathcal{V}^{*}$. Therefore, it is possible to select a full column rank matrix $V$ forming a basis of $\mathcal{V}^{*}$ adapted to $\mathcal{N}$, i.e.

$$
V=\left[\begin{array}{ll}
V_{\overline{\mathcal{N}}}, & N
\end{array}\right] .
$$

Define $n_{\mathcal{N}}=\operatorname{dim}(\mathcal{N})$. Then, $V_{\mathcal{N}} \in \Re^{n \times\left(n-n_{\mathcal{V}}-n_{\mathcal{N}}\right)}$ and $N \in \Re^{n \times n_{\mathcal{N}}}$. Moreover, matrix $V$ satisfied the following equalities

$$
\begin{gathered}
A V+E K^{*}=V Q \Leftrightarrow\left(A+E \bar{K}^{*}\right) V=V Q, \\
C V=0,
\end{gathered}
$$

for some matrices $\bar{K}^{*} \in \Re^{m \times n}, K^{*} \in \Re^{m \times\left(n-n_{\mathcal{V}}\right)}$ and $Q \in \Re^{\left(n-n_{\mathcal{V}}\right) \times\left(n-n_{\mathcal{V}}\right)}$. Notice that (13)-(14) are the matrix representations of the map (5), and that $V^{+} V=I$ implies $\bar{K}^{*}=K^{*} V^{+}$, satisfying (13).

The following nonsingular transformation matrix can be defined:

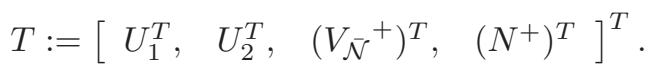

The transformation $\bar{x}(t)=T x(t)$, with matrix $T$ designed according to (15), transforms the system (1) into the following form:

$$
\begin{aligned}
& {\left[\begin{array}{c}
\overline{\bar{x}}_{11}(t) \\
\overline{\bar{x}}_{12}(t) \\
\overline{\bar{x}}_{21}(t) \\
\overline{\bar{x}}_{22}(t)
\end{array}\right]=\left[\begin{array}{cccc}
A_{11} & A_{12} & 0 & 0 \\
A_{21} & A_{22} & 0 & 0 \\
A_{31} & A_{32} & A_{33} & 0 \\
A_{41} & A_{42} & A_{43} & A_{44}
\end{array}\right]\left[\begin{array}{c}
\bar{x}_{11}(t) \\
\bar{x}_{12}(t) \\
\bar{x}_{21}(t) \\
\bar{x}_{22}(t)
\end{array}\right]+\left[\begin{array}{c}
B_{1} \\
B_{2} \\
B_{3} \\
B_{4}
\end{array}\right] u(t)+\left[\begin{array}{c}
0 \\
E_{12} \\
E_{21} \\
E_{22}
\end{array}\right] \bar{f}(t),} \\
& y(t)=C_{1}\left[\bar{x}_{11}(t)^{T}, \quad \bar{x}_{12}(t)^{T}\right]^{T}, \\
& \bar{f}(t)=f(t)-K_{1}^{*} \bar{x}_{21}(t),
\end{aligned}
$$

where $\bar{x}_{11}(t) \in \Re^{n_{\mathcal{V}}-m}, \bar{x}_{12}(t) \in \Re^{m}, \bar{x}_{21}(t) \in \Re^{n-n_{\mathcal{V}}-n_{\mathcal{N}}}, \bar{x}_{22}(t) \in \Re^{n_{\mathcal{N}}}, K_{1}^{*} \in \Re^{m \times\left(n-n_{\mathcal{V}}-n_{\mathcal{N}}\right)}$ and

$$
\begin{gathered}
{\left[\begin{array}{cccc}
A_{11} & A_{12} & 0 & 0 \\
A_{21} & A_{22} & 0 & 0 \\
A_{31} & A_{32} & A_{33} & 0 \\
A_{41} & A_{42} & A_{43} & A_{44}
\end{array}\right]=T\left(A+E \bar{K}^{*}\right) T^{-1}, \quad\left[\begin{array}{c}
0 \\
E_{12} \\
E_{21} \\
E_{22}
\end{array}\right]=T E,} \\
C_{1}=C\left[\left(U_{1}^{+}\right)^{T}, \quad\left(U_{2}^{+}\right)^{T}\right]^{T},
\end{gathered}
$$




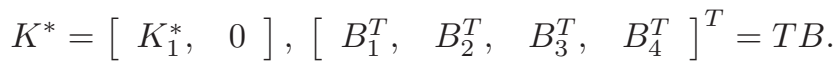

For the system (16)-(18) it is possible to demonstrate that the set of invariant zeroes that do not belong to unobservable subspace $\mathcal{N}$ is equal to the set of eigenvalues of the matrix $A_{33}$, and, the set of invariant zeroes that belong to unobservable subspace $\mathcal{N}$ is equal to the set of eigenvalues of the matrix $A_{44}$ (see, for example [52]). Notice that this transformation should be computed for each operating mode $j \in \mathcal{Q}$.

\section{OBSERVER DESIGN}

The continuous and discrete state observers have been previously presented in [1]. However, in this version, the detailed proofs of the theorems are described.

Consider the state estimation problem for a constant index $j(x(t))=j^{*}=$ const. Let us describe the observer design for each partition of the state $\bar{x}(t)$.

\subsection{State Observer for $\bar{x}_{11}(t)$ and $\bar{x}_{12}(t)$}

Consider the first two parts of the system (16)-(18) for $j(x(t))=j^{*}$ with the partition $\bar{x}_{1}(t)=$ $\left[\bar{x}_{11}(t)^{T}, \bar{x}_{12}(t)^{T}\right]^{T}$, i.e.

$$
\begin{aligned}
& \dot{\bar{x}}_{1}(t)=A_{1 j^{*}} \bar{x}_{1}(t)+E_{1 j^{*}} \bar{f}_{1}(t)+B_{12 j^{*}} u(t), \\
& y(t)=C_{1 j^{*}} \bar{x}_{1}(t),
\end{aligned}
$$

where

$$
A_{1 j^{*}}=\left[\begin{array}{ll}
A_{11 j^{*}} & A_{12 j^{*}} \\
A_{21 j^{*}} & A_{22 j^{*}}
\end{array}\right], E_{1 j^{*}}=\left[\begin{array}{c}
0 \\
E_{12 j^{*}}
\end{array}\right], B_{12 j^{*}}=\left[\begin{array}{c}
B_{1 j^{*}} \\
B_{2 j^{*}}
\end{array}\right] .
$$

In accordance with the structure of the transformation, the states $\bar{x}_{1}(t)$ forms the strongly observable subspace. Then, the following observer for $\bar{x}_{1}(t)$ could be designed (see [31])

$$
\begin{aligned}
& \hat{x}_{1_{j^{*}}}(t)=z_{1_{j^{*}}}(t)+P_{1_{j^{*}}}^{-1} \nu_{j^{*}}(t), \\
& \dot{z}_{1_{j^{*}}}(t)=A_{1 j^{*}} z_{1 j^{*}}(t)+B_{12 j^{*}} u(t)+L_{1 j^{*}}\left(y(t)-C_{1 j^{*}} z_{1 j^{*}}(t)\right), \\
& \dot{\nu}_{j^{*}}(t)=W_{j^{*}}\left(y(t)-C_{1 j^{*}} z_{1 j^{*}}(t), \nu_{j^{*}}(t)\right),
\end{aligned}
$$

where $z_{1^{*}}(t), \hat{x}_{1^{*}}(t) \in \Re^{n \mathcal{V}_{j^{*}}}$ and the matrix $L_{1 j^{*}} \in \Re^{n \mathcal{V}_{j^{*}} \times m}$ is selected such that matrix $\left(A_{1 j^{*}}-L_{1 j^{*}} C_{1 j^{*}}\right)=A_{L_{1 j^{*}}}$ is Hurwitz ${ }^{\S}$. The distribution matrix $P_{1 j^{*}}$ takes the following structure

$$
\begin{aligned}
& P_{1 j^{*}}=\left[\begin{array}{lll}
c_{1 j^{*}}^{T}, & \left(c_{1 j^{*}} A_{L_{1} j^{*}}\right.
\end{array}\right)^{T}, \quad \cdots, \quad\left(c_{1 j^{*}} A_{L_{1 j^{*}}}^{r_{1 j^{*}}}-1\right)^{T}, \quad \cdots, \\
& \left.c_{m_{j^{*}}}^{T}, \quad\left(c_{m_{j^{*}}} A_{L_{1 j^{*}}}\right)^{T}, \quad \cdots, \quad\left(c_{m_{j^{*}}} A_{L_{1} j^{*}}^{r_{m^{*}}-1}\right)^{T}\right]^{T} .
\end{aligned}
$$

According to Assumption 2 the condition $\operatorname{rank}\left(P_{1 j^{*}}\right)=n_{\mathcal{V}_{j^{*}}}$ is satisfied. The vector $\nu_{j^{*}}(t)=$

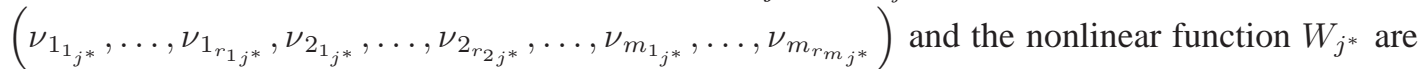
chosen using the HOSM differentiator (for more details, see [53])

$$
\begin{aligned}
& \dot{\nu}_{k_{j^{*}}}=\nu_{k_{j^{*}}}-\alpha_{k_{j^{*}}} M_{k_{j^{*}}}(t)^{\frac{1}{r_{k^{*}}}}\left[\nu_{k_{j^{*}}}-e_{y_{k^{*}}}\right\rfloor^{\frac{r_{k j^{*}}-1}{r_{k j^{*}}}}, \\
& \dot{\nu}_{k_{i_{j}}}=\nu_{k_{(i+1)} j^{*}}-\alpha_{k_{i_{j^{*}}}} M_{k_{j^{*}}}(t)^{\frac{1}{r_{k j^{*}}-i+1}}\left[\nu_{k_{i^{*}}}-\dot{\nu}_{k_{(i-1) j^{*}}}\right\rfloor^{\frac{r_{k j^{*}-i}}{r_{k^{*}}-i+1}}, i=2, \ldots, r_{k^{*}}-1 \text {, } \\
& \dot{\nu}_{k_{r_{k^{*}}}}=-\alpha_{k_{r_{k^{*}}}} M_{k_{j^{*}}}(t)\left[\nu_{k_{r_{k^{*}}}}-\dot{\nu}_{k_{r_{k^{*}}-1}}\right]^{0}, \forall k=1, \ldots, m \text {, }
\end{aligned}
$$

$\bar{\S}$ Due to Assumption 2 and Definition 6 such matrix $L_{1} j^{*}$ always exist. 
where $e_{y_{j^{*}}}(t)=y_{k}(t)-c_{k} z_{1 j^{*}}(t)$, and the constants $\alpha_{k_{i_{j^{*}}}}$ are chosen recursively and sufficiently large. In particular, according to [54], one possible choice is $\alpha_{k_{j^{*}}}=1.1, \alpha_{k_{j^{*}}}=1.5, \alpha_{k_{j_{j^{*}}}}=2$, $\alpha_{k_{j^{*}}}=3, \alpha_{k_{j^{*}}}=5, \alpha_{k_{j^{*}}}=8$, which is enough for the case that $r_{k^{*}} \leq 6, \forall k=1, \ldots, m$.

Remark 2: According to [54], if $y_{k}(t)$ is a $\left(r_{k j^{*}}\right)$-times continuously differentiable signal with a bounded Lebesgue measurable noise $v_{k}(t) \in \mathcal{L}_{\infty}$. Then, there exist $0 \leq t_{j^{*}}<\infty$ and constants $\mu_{k_{i_{j}}}$, only dependent on $\alpha_{k_{i^{*}}}$ and $M_{k_{j^{*}}}$, such that for all $t \geq t_{j^{*}}$, the $\nu_{k_{i_{j^{*}}}}$ in (24) satisfy

$$
\left|\nu_{k_{(i+1)} j^{*}}-e_{y_{k_{j^{*}}}}^{(i)}\right| \leq \mu_{k_{i_{j^{*}}}}\left\|v_{k}\right\|_{\infty}^{\frac{r_{k j^{*}-i+1}}{r_{k j^{*+1}}}}, \forall i=0, \ldots, r_{k j^{*}}, \forall k=1, \ldots, m
$$

The continuous functions $M_{k_{j^{*}}}(t)$ are known locally Lipschitz constants, at time $t$, for each $e_{y_{k_{j^{*}}}}^{\left(r_{k_{j^{*}}}\right)}$, and they can be computed in the following way.

Proposition 1: There exist a time $\tilde{t}$ and known positive functions $\beta_{k_{1 j^{*}}}(t)$, and constants $\beta_{k_{2 j^{*}}}$, $\beta_{k_{3 j^{*}}}, k_{L_{j^{*}}}$ and $\lambda_{L_{1 j^{*}}}$ such that

$$
\left|e_{y_{j^{*}}}^{\left(r_{k_{j}}\right)}\right| \leq M_{k_{j^{*}}}(t), \quad \forall t>\tilde{t}
$$

with

$$
M_{k_{j^{*}}}(t)=\beta_{k_{1 j^{*}}}(t)+\beta_{k_{2 j^{*}}} \int_{0}^{t} \exp \left(-\lambda_{L_{1 j^{*}}}(t-\tau)\right)\|\bar{f}(\tau)\| d \tau+\beta_{k_{3 j^{*}}}, \forall k=1, \ldots, m
$$

Proof

Due to matrix $A_{L_{1} j^{*}}$ is Hurwitz, there exist known positive constants $k_{L_{1_{j}}}, \lambda_{L_{1 j^{*}}}$ such that $\left\|\exp \left(A_{L_{1 j^{*}}} t\right)\right\| \leq k_{L_{1} j^{*}} \exp \left(-\lambda_{L_{1 j^{*}}} t\right)$. Therefore, from the solution of the estimation error for observer (21), i.e. $\bar{e}_{1_{j^{*}}}(t)=\bar{x}_{1}(t)-z_{1 j^{*}}(t)$, see (27), it is obtained

$$
\left\|\bar{e}_{1_{j^{*}}}(t)\right\| \leq k_{L_{j^{*}}} \exp \left(-\lambda_{L_{1 j^{*}}} t\right)\left\|\bar{e}_{1_{j^{*}}}(0)\right\|+\left\|E_{1_{j^{*}}}\right\| \int_{0}^{t} \exp \left(-\lambda_{L_{1 j^{*}}}(t-\tau)\right)\|\bar{f}(\tau)\| d \tau,
$$

For an arbitrary constant $\lambda_{L_{1 j^{*}}}$, there exists a time $\tilde{t}$ such that $k_{L_{1^{*}}} \exp \left(-\lambda_{L_{1 j^{*}}} t\right)\left\|\bar{e}_{1_{j^{*}}}(0)\right\|$ is less than $\Gamma_{j^{*}}(t), \forall t \geq \tilde{t}$. Thus

$$
\left\|\bar{e}_{1_{j^{*}}}(t)\right\| \leq \Gamma_{j^{*}}(t)+k_{L_{j^{*}}}\left\|E_{1_{j^{*}}}\right\| \int_{0}^{t} \exp \left(-\lambda_{L_{1 j^{*}}}(t-\tau)\right)\|\bar{f}(\tau)\| d \tau
$$

From above inequality and the output error dynamics $e_{y_{k^{*}}}^{\left(r_{k^{*}}\right)}(t)$, see (30), the following inequality is obtained

$$
\begin{array}{r}
\left|e_{y_{k^{*}}}^{\left(r_{k_{j^{*}}}\right)}(t)\right| \leq\left|c_{k}\right|\left\|A_{L_{1 j^{*}}}^{r_{k_{j^{*}}}}\right\|\left(\Gamma_{j^{*}}(t)+k_{L_{j^{*}}}\left\|E_{1_{j^{*}}}\right\| \int_{0}^{t} \exp \left(-\lambda_{L_{1 j^{*}}}(t-\tau)\right)\|\bar{f}(\tau)\|\right) \\
+\left|c_{k}\right|\left\|A_{L_{1 j^{*}}}^{r_{k_{j^{*}}}-1}\right\|\left\|E_{1_{j^{*}}}\right\|\|\bar{f}(t)\| .
\end{array}
$$

Thus, Proposition 1 is proven with $\beta_{k_{1 j^{*}}}(t)=\left|c_{k}\right|\left\|A_{L_{1} j^{*}}^{r_{k^{*}}}\right\| \Gamma_{j^{*}}(t), \quad \beta_{k_{2 j^{*}}}=$ $k_{L_{1^{*}}}\left|c_{k}\right|\left\|A_{L_{1} j^{*}}^{r_{k_{j *}}}\right\|\left\|E_{1_{j^{*}}}\right\|$, and $\beta_{k_{3 j^{*}}}=\left|c_{k}\right|\left\|A_{L_{1} j^{*}}^{r_{k_{j^{*}}}-1}\right\|\left\|E_{1_{j^{*}}}\right\|\|\bar{f}(t)\|$.

Taking into account the previous explanations, the following theorem can be stated. 
Theorem 2: Let $j(x(t))=j^{*}=$ const, and the observer (20)-(22) with the correction terms (24) be applied to the system (19). Let Assumptions 1 - 2 be satisfied. Then, provided that constants $\alpha_{k_{i}}$ are chosen properly and $M_{k_{j^{*}}}(t)$ are selected as in Proposition 1 , the state estimation error for $\bar{x}_{1}(t)$ converges to zero exactly and in a finite time, i.e. $e_{1_{j^{*}}}(t)=\bar{x}_{1}(t)-\hat{x}_{1_{j^{*}}}(t)=0 \forall t \in\left[t_{j^{*}}, t_{1}\right)^{\top}$.

Proof

Let us define the estimation $\bar{e}_{1^{*}}(t)=\bar{x}_{1}(t)-z_{1 j^{*}}(t)$. The dynamics of $\bar{e}_{1_{j^{*}}}(t)$ is given by:

$$
\begin{aligned}
\dot{\bar{e}}_{1_{j^{*}}}(t) & =A_{L_{1 j^{*}}} \bar{e}_{1_{j^{*}}}(t)+E_{1 j^{*}} \bar{f}(t), \\
e_{y_{j^{*}}}(t) & =C_{1 j^{*}} e_{y_{j^{*}}}(t) .
\end{aligned}
$$

Due to Assumption 2 it is always possible to compute matrix $L_{1 j^{*}}$ such that the matrix $A_{L_{1} j^{*}}$ is Hurwitz.

Applying the transformation $P_{1 j^{*}}$ to the estimation error $\bar{e}_{1_{j^{*}}}$, the system (27) can be transformed into

$$
P_{1 j^{*}} \dot{\bar{e}}_{j^{*}}(t)=P_{1 j^{*}} A_{L_{1 j^{*}}} \bar{e}_{j_{j^{*}}}(t)+P_{1 j^{*}} E_{1 j^{*}} \bar{f}(t) .
$$

According to Assumption 2 and the structure of the transformation, (29) can be rewritten as

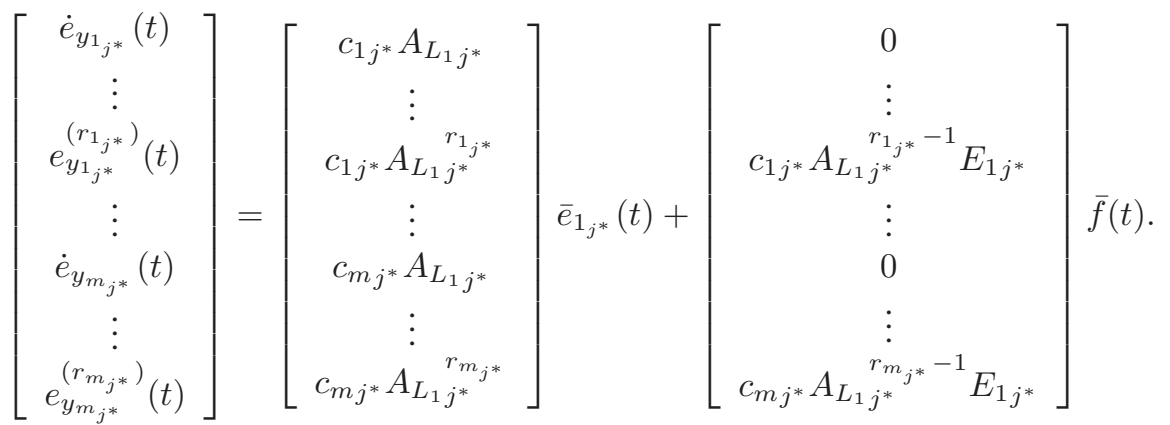

Notice that the derivatives of $e_{y_{k^{*}}}(t)$ are estimated by the HOSM differentiator (24). Thus, if $M_{k_{j^{*}}}(t)$ are selected as in Proposition 1, the differentiator converges (for more details see [53]), therefore

$$
P_{1^{*}} \bar{e}_{1_{j^{*}}}(t)=\nu_{j^{*}}(t) .
$$

The estimation of the variable $\bar{x}_{1}(t)$ is obtained by algebraic manipulation as

$$
\hat{x}_{1_{j^{*}}}(t)=z_{1 j^{*}}(t)+P_{1^{*}}^{-1} \nu_{j^{*}}(t) .
$$

Therefore, the exact convergence to zero for $e_{1_{j^{*}}}(t)=\bar{x}_{1}(t)-\hat{x}_{1_{j^{*}}}(t)$ is obtained. Notice that if there exists measurement noise, the state estimation error order will be directly proportional to order of the noise, as Remark 2 shows.

Remark 3: Notice that it is possible to consider, in addition to the faults $f(t)$, disturbances with the same structural properties in its distribution matrix, i.e. $\bar{f}(t)=f(t)+d(t)-K_{1}^{*} \bar{x}_{21}(t)$. The observer (20)-(22) will keep on estimating exactly and in finite time the state $\bar{x}_{1}$ provided that Proposition 1 is satisfied (taking into account bounded disturbances, i.e. $\|d(t)\| \leq d^{+}<\infty$, with a known constant $d^{+}$).

$\overline{{ }^{\top}} t_{j *}$ is the time when the observer (20)-(22) has converged to zero, and $t_{1}$ is the first switching time. 


\subsection{State Observer for $\bar{x}_{21}(t)$}

Let $\hat{x}_{21_{j^{*}}}(t)$ be the state observer for $\bar{x}_{21}(t)$ defined by

$$
\begin{aligned}
\hat{x}_{21_{j^{*}}}(t) & =z_{2 j^{*}}(t)+L_{2 j^{*}} \hat{x}_{12_{j^{*}}}(t), \\
\dot{z}_{2_{j^{*}}}(t) & =\bar{A}_{1_{j^{*}}} \hat{x}_{11_{j^{*}}}(t)+\bar{A}_{2_{j^{*}}} \hat{x}_{12_{j^{*}}}(t)+A_{L_{2 j^{*}}} \hat{x}_{21_{j^{*}}}(t)+\bar{B}_{2_{j^{*}}} u(t),
\end{aligned}
$$

where the estimations of $\hat{x}_{11_{j^{*}}}(t)$ and $\hat{x}_{12_{j^{*}}}(t)$ are provided by observer (20)-(22). The matrices in (34) are defined by

$$
\begin{aligned}
\bar{A}_{1_{j^{*}}} & =A_{31 j^{*}}-L_{2 j^{*}} A_{21 j^{*}}, \\
\bar{A}_{2_{j^{*}}} & =A_{32 j^{*}}-L_{2 j^{*}} A_{22 j^{*}}, \\
A_{L_{2 j^{*}}} & =A_{33 j^{*}}-E_{21 j^{*}} K_{1 j^{*}}^{*}+L_{2 j^{*}} E_{12 j^{*}} K_{1 j^{*}}^{*}, \\
\bar{B}_{2_{j^{*}}} & =B_{3 j^{*}}-L_{2 j^{*}} B_{2 j^{*}} .
\end{aligned}
$$

Then, the following theorem can be stated.

Theorem 3: Let $j(x(t))=j^{*}=$ const be satisfied. Then, provided that the matrix $L_{2} j^{*}$ is selected such that the matrix $A_{L_{2} j^{*}}$ is Hurwitz, the state estimation error for $\bar{x}_{21}(t)$ is ultimately bounded by a constant $\gamma_{j^{*}} f^{+}$, i.e. $\left\|e_{21_{j^{*}}}(t)\right\|=\left\|\bar{x}_{21}(t)-\hat{x}_{21_{j^{*}}}(t)\right\| \leq \gamma_{j^{*}} f^{+}$as $t \rightarrow \infty$.

Proof

From the dynamics $\dot{\bar{x}}_{12}(t)$ in (16) it is obtained

$$
E_{12 j^{*}} K_{1 j^{*}}^{*} \bar{x}_{21}(t)=\left(A_{21 j^{*}} \bar{x}_{11}(t)+A_{22 j^{*}} \bar{x}_{12}(t)+B_{2 j^{*}} u(t)+E_{12 j^{*}} f(t)\right)-\dot{\bar{x}}_{12}(t) .
$$

Adding and subtracting $L_{2 j^{*}} E_{12 j^{*}} K_{1 j^{*}}^{*} \bar{x}_{21}(t)$ in the dynamics $\dot{x}_{21}(t)$ it is obtained

$$
\dot{\bar{x}}_{21}(t)=\bar{A}_{1_{j^{*}}} \bar{x}_{11}(t)+\bar{A}_{2_{j^{*}}} \bar{x}_{12}(t)+A_{L_{2} j^{*}} \bar{x}_{21}(t)+\bar{B}_{2_{j^{*}}} u+E_{L_{2} j^{*}} f(t)+L_{2 j^{*}} \dot{\bar{x}}_{21}(t),
$$

where $E_{L_{2} j^{*}}=E_{21 j^{*}}-L_{2 j^{*}} E_{12 j^{*}}$.

The dynamics error $e_{21_{j^{*}}}(t)=\bar{x}_{21}(t)-\hat{x}_{21_{j^{*}}}(t)$ is governed by

$$
\dot{e}_{21_{j^{*}}}(t)=A_{L_{2} j^{*}} e_{21_{j^{*}}}(t)+E_{L_{2} j^{*}} f(t) .
$$

Thus, $L_{2 j^{*}}$ has to be selected such that the matrix $A_{L_{2} j^{*}}$ is Hurwitz. Finally, it is clear that $e_{21_{j^{*}}}(t)$ is bounded by

$$
\left\|e_{21_{j^{*}}}(t)\right\| \leq \gamma_{j^{*}} f^{+} \text {, as } t \rightarrow \infty
$$

with $\gamma_{j^{*}}=\frac{k_{L_{2 j^{*}}}\left\|E_{L_{2 j^{*}}}\right\|}{\lambda_{L_{2 j^{*}}}}$, and the positive constants $k_{L_{2 j^{*}}}, \lambda_{L_{2 j^{*}}}$ such that $\left\|\exp \left(A_{L_{2 j^{*}}} t\right)\right\| \leq$ $k_{L_{2 j^{*}}} \exp \left(-\lambda_{L_{2 j^{*}}} t\right)$.

Such matrix $L_{2 j^{*}}$ always exist if the pair $\left(E_{12 j^{*}} K_{1 j^{*}}^{*}, A_{33 j^{*}}-E_{21 j^{*}} K_{1 j^{*}}\right)$ is detectable. To prove this consider the Rosenbrock matrix of system (16)-(17), without faults, i.e.

$$
R(s)=\left[\begin{array}{cc|c|c}
s I-A_{11 j^{*}} & -A_{12 j^{*}} & 0 & 0 \\
C_{11 j^{*}} & C_{12 j^{*}} & 0 & 0 \\
-A_{21 j^{*}} & s I-A_{22 j^{*}} & E_{12 j^{*}} K_{1 j^{*}} & 0 \\
-A_{31 j^{*}} & -A_{32 j^{*}} & s I-A_{33 j^{*}}+E_{21 j^{*}} K_{1 j^{*}} & 0 \\
-A_{41 j^{*}} & -A_{42 j^{*}} & -A_{43 j^{*}} & s I-A_{44 j^{*}}
\end{array}\right] .
$$

Assumption 1 implies that $A_{44 j^{*}}$ is Hurwitz and the rank of matrix $R(s)$ is full except for $s$ being an eigenvalue of $A_{44 j^{*}}$. Thus, it is clear that rank of the first column is equal to $n_{\mathcal{V}^{*}}$ since the pair $\left(C_{1 j^{*}}, A_{1 j^{*}}\right)$ is observable; rank of the third column is equal to $n_{\mathcal{N}_{j^{*}}}$ due to Assumption 1; then, rank of the second column is equal to $n-n_{\mathcal{V}_{j^{*}}}-n_{\mathcal{N}_{j^{*}}}$ implying that the pair $\left(E_{12 j^{*}} K_{1 j^{*}}^{*}, A_{33 j^{*}}-E_{21 j^{*}} K_{1 j^{*}}^{*}\right)$ is detectable and that matrix $L_{2 j^{*}}$ always exists. 
Remark 4: If there exists disturbances $E_{L_{2} j^{*}} d(t)$, the state estimation error $e_{21_{j^{*}}}(t)$ will be bounded by $\left\|e_{21_{j^{*}}}(t)\right\| \leq \gamma_{j^{*}} f^{+}+\varsigma_{j^{*}} d^{+}$, as $t \rightarrow \infty$, with $\varsigma_{j^{*}}$ positive known constant.

Remark 5: It is possible to formulate a constrained linear optimization problem in order to select the matrix $L_{2 j^{*}}$ in such a way that the estimation error $e_{21_{j^{*}}}(t)$ is minimized.

\subsection{State Observer for $\bar{x}_{22}(t)$}

Without loss of generality it is assumed that

$$
\operatorname{rank}\left[\begin{array}{c}
E_{12 j^{*}} \\
E_{21 j^{*}}
\end{array}\right]=\operatorname{rank}\left(E_{2 j^{*}}\right)=m
$$

Let $\hat{x}_{22_{j^{*}}}(t)$ be the state observer for $\bar{x}_{22}(t)$ defined by

$$
\begin{aligned}
& \hat{x}_{22_{j^{*}}}(t)=z_{3 j^{*}}(t)+E_{22 j^{*}} E_{2 j^{*}}^{+}\left[\begin{array}{c}
\hat{x}_{12}(t) \\
\hat{x}_{21}(t)
\end{array}\right], \\
& \dot{z}_{3_{j^{*}}}(t)=A_{41_{j^{*}}} \hat{x}_{11_{j^{*}}}(t)+A_{42_{j^{*}}} \hat{x}_{12_{j^{*}}}(t)+A_{43_{j^{*}}} \hat{x}_{21_{j^{*}}}(t)+A_{44_{j^{*}}} \hat{x}_{22_{j^{*}}}+B_{4 j^{*}} u_{j^{*}}(t) \\
& -E_{22 j^{*}} E_{2}+{ }_{j^{*}}^{+}\left[\begin{array}{c}
\left(A_{21_{j^{*}}} \hat{x}_{11_{j^{*}}}(t)+A_{22_{j^{*}}} \hat{x}_{12_{j^{*}}}(t)\right. \\
\left.+B_{2_{j^{*}}} u(t)\right) \\
\left(A_{31_{j^{*}}} \hat{x}_{11_{j^{*}}}(t)+A_{3 j_{j^{*}}} \hat{x}_{12_{j^{*}}}(t)\right. \\
\left.+A_{33_{j^{*}}} \hat{x}_{21_{j^{*}}}(t)+B_{3_{j^{*}}} u(t)\right)
\end{array}\right],
\end{aligned}
$$

where the estimations of $\hat{x}_{11_{j^{*}}}(t)-\hat{x}_{12_{j^{*}}}(t)$ and $\hat{x}_{21_{j^{*}}}(t)$ are provided by observers (20)-(22) and (33)-(34), respectively.

Then, the following theorem can be stated.

Theorem 4: Let $j(x(t))=j^{*}=$ const and $\operatorname{rank}\left(E_{2 j^{*}}\right)=m$ be satisfied. Then, the state estimation error for $\bar{x}_{22}(t)$ is ultimately bounded by a constant $\delta_{j^{*}} f^{+}$, i.e. $\left\|e_{22_{j^{*}}}(t)\right\|=\left\|\bar{x}_{22}(t)-\hat{x}_{22_{j^{*}}}(t)\right\| \leq$ $\delta_{j^{*}} f^{+}$as $t \rightarrow \infty$.

Proof

Since $\operatorname{rank}\left(E_{2 j^{*}}\right)=m$, from (16), $\bar{f}(t)$ can be rewritten as

$$
\bar{f}(t)=E_{22 j^{*}} E_{2}{ }_{j^{*}}^{+}\left[\begin{array}{c}
\dot{\bar{x}}_{12}(t)-\left(A_{21_{j^{*}}} \bar{x}_{11}(t)+A_{22_{j^{*}}} \bar{x}_{12}(t)\right. \\
\left.+B_{2_{j^{*}}} u(t)\right) \\
\dot{\bar{x}}_{21}(t)-\left(A_{31_{j^{*}}} \bar{x}_{11}(t)+A_{32_{j^{*}}} \bar{x}_{12}(t)\right. \\
\left.+A_{33_{j^{*}}} \bar{x}_{21}(t)+B_{3_{j^{*}}} u(t)\right)
\end{array}\right] .
$$

The dynamics error $e_{22_{j^{*}}}(t)=\bar{x}_{22}(t)-\hat{x}_{22_{j^{*}}}(t)$, substituting $\bar{f}(t)$, is governed by

$$
\dot{e}_{22_{j^{*}}}(t)=A_{44 j^{*}} e_{22_{j^{*}}}(t)+A_{43 j^{*}} e_{21_{j^{*}}}(t) .
$$

Since $A_{44 j^{*}}$ is Hurwitz, using (42), the error $e_{22_{j^{*}}}(t)$ is bounded by

$$
\left\|e_{22_{j^{*}}}(t)\right\| \leq \delta_{j^{*}} f^{+} \text {, as } t \rightarrow \infty
$$

with $\delta_{j^{*}}=\frac{k_{44} j^{*}\left\|A_{43 j^{*}}\right\|}{\lambda_{44} j_{j^{*}}} \gamma_{j^{*}}$, and the positive constants $k_{44_{j^{*}}}, \lambda_{44_{j^{*}}}$ such that $\left\|\exp \left(A_{44 j^{*}} t\right)\right\| \leq$ $k_{44_{j^{*}}} \exp \left(-\lambda_{44_{j^{*}}} t\right)$.

Remark 6: If there exists disturbances $E_{22 j^{*}} d(t)$, the state estimation error $e_{22_{j^{*}}}(t)$ will be bounded by $\left\|e_{22_{j^{*}}}(t)\right\| \leq \delta_{j^{*}} f^{+}+\varrho_{j^{*}} d^{+}$, as $t \rightarrow \infty$, with $\varrho_{j^{*}}$ positive known constant. 


\subsection{Bank of Observers}

To solve the continuous state estimation problem for the NMP system (1), the following bank of observers is proposed

$$
\begin{aligned}
& \tilde{x}_{1_{\lambda}}(t)=z_{1 \lambda}(t)+P_{1 \lambda}^{-1} \nu_{\lambda}(t), \\
& \tilde{x}_{21_{\lambda}}(t)=z_{2 \lambda}(t)+L_{2 \lambda} \tilde{x}_{12_{\lambda}}(t), \\
& \tilde{x}_{22_{\lambda}}(t)=z_{3 \lambda}(t)+E_{22 \lambda} E_{2}+\left[\begin{array}{c}
\tilde{x}_{12}(t) \\
\tilde{x}_{21}(t)
\end{array}\right], \\
& \tilde{y}(t)=C_{1 \lambda} \tilde{x}_{1}(t), \forall \lambda=1, \ldots, q,
\end{aligned}
$$

where $\tilde{x}_{1_{\lambda}}(t), \tilde{x}_{21_{\lambda}}(t), \tilde{x}_{22_{\lambda}}(t)$ and theirs components are designed according to Section 4. Now, the following assumption is stated.

Assumption 3: Assume that the initial discrete state is known.

Theorems 2, 3 and 4 establish that the $j^{*}$ observer reconstructs the continuous state correctly, and according to Assumption 3 we know the $j^{*}$ observer has made it on the interval $\left[0, t_{1}\right)$. According to [55], to know when the $j^{*}$ observer has converged it is sufficient to verify that the following inequality is satisfied

$$
\left\|e_{\tilde{y}_{j^{*}}}(t)\right\| \leq \xi_{j^{*}} M_{j^{*}}^{+} h^{n_{\mathcal{V}_{j *}}}, \forall t \in\left[t_{1}-\xi_{j^{*}} h, t_{1}\right)
$$

where $e_{\tilde{y}_{j^{*}}}=y-\tilde{y}_{j^{*}}, \xi_{j^{*}}$ and $\xi_{j^{*} t}$ are positive constants, $h$ is the sampling time and $M_{j^{*}}^{+}=$ $\max _{\forall k=1, \ldots, m}\left(M_{k_{j^{*}}}(t)\right)$. It is natural to estimate the constants $\xi_{i^{*}}$ and $\xi_{i^{*}}$ through simulation. Thus, in this way it is possible to determine when the $j^{*}-t h$ observer has converged to the correct continuous state during the time interval $t \in\left[0, t_{1}\right)$.

Then, the real estimated state $\hat{x}$ is defined as follows:

$$
\hat{x}=\tilde{x}_{j^{*}}, \quad \forall t \in\left[t_{j^{*}}, t_{1}\right) . \|
$$

Due to the transformation $\bar{x}(t)=T x(t)$, the bank of observers for the original state vector has to be designed as:

$$
\hat{x}(t)=T_{\lambda}^{-1}\left[\begin{array}{lll}
\tilde{x}_{11_{\lambda}}^{T}(t), & \tilde{x}_{12_{\lambda}}^{T}(t), \quad \tilde{x}_{21_{\lambda}}^{T}(t), \quad \tilde{x}_{22_{\lambda}}^{T}(t)
\end{array}\right]^{T} .
$$

Theorem 5: The original state estimation error generated by the observer (53) and system (1) is ultimately bounded by a positive constant $\rho_{\lambda} f^{+}$, i.e.

$$
\|\hat{x}(t)-x(t)\| \underset{t \rightarrow \infty}{\leq} \rho_{\lambda} f^{+}
$$

Proof

It is clear from Theorems (2), (3) and (4), and the coordinates combination that the error generated by the estimation properties in the transformed coordinates is propagated in the original coordinates, producing $\|\hat{x}(t)-x(t)\| \leq \rho_{\lambda} f^{+}$with a positive constant $\rho_{\lambda}$ defined by $\delta_{\lambda}$.

\section{DISCRETE STATE ESTIMATION}

Once the continuous state is estimated, the following discrete state observer is proposed:

$$
\lambda(\hat{x})=\left\{\begin{array}{ccc}
1, & \forall \hat{x} \mid & H \hat{x} \in \mathcal{H}_{1} \\
2, & \forall \hat{x} \mid & H \hat{x} \in \mathcal{H}_{2} \\
\vdots & & \\
q, & \forall \hat{x} \mid & H \hat{x} \in \mathcal{H}_{q}
\end{array},\right.
$$

\footnotetext{
"Notice that it is always possible to design the gain $M_{j^{*}}(t)$ in such a way that inequality $t_{j^{*}}<t_{1}$ is satisfied.
} 
For each discrete state $j$ define the observability matrix of the discrete state as

$$
Q_{j}=\left[\begin{array}{llll}
H^{T}, & \left(H A_{j}\right)^{T}, & \cdots, & \left(H A_{j}^{n-1}\right)^{T}
\end{array}\right]^{T} .
$$

The exactly and finite time discrete state estimation is described by the following lemma.

Lemma 6: Under the statement of Theorem 2, the discrete state $j(x(t))$ is estimated exactly and in finite time if the following condition is satisfied

$$
\mathcal{V}_{j}^{*} \subset \operatorname{ker}\left(Q_{j}\right), \forall j=1, \ldots, q,
$$

where $\mathcal{V}_{j}^{*}$ is the weakly unobservable subspace for each $j$.

Proof

If (56) is satisfied, then there exist a matrix $P_{Q_{j}} \in \Re^{n \times n v_{j}}$ such that $H x(t)=H T_{j} P_{Q_{j}} \bar{x}_{1}(t)$. According to exact and finite time convergence properties of $\hat{x}_{1}(t)$ described in Section 4.1 Theorem 2, the discrete state can be estimated exactly and in finite time by means of (55) with $H x(t)=H T_{j} P_{Q_{j}} \bar{x}_{1}(t)$.

Remark 7: Condition (56) implies that an exact estimation of the discrete state is achieved only if the scalar function $j(x(t))$ can be represented as a combination of the strongly observable states, i.e. $\bar{x}_{11}(t)$ and $\bar{x}_{12}(t)$, as a consequence of the observer properties described by the Theorem 2.

\subsection{State Estimation On Switching Times}

Let $t_{i}{ }^{+}$be the time instants after the switching times $t_{i}$. In order to maintain the state estimation on the switching times the following algorithm is proposed.

Proposition 7: The state estimation of system (1) is maintained in spite of the switchings if the following reset equations are implemented in the bank of observers (47)-(50) for all $\lambda \neq j^{*}$

$$
\begin{aligned}
\nu_{\lambda}\left(t_{i}{ }^{+}\right) & =0 \\
z_{1_{\lambda}}\left(t_{i}{ }^{+}\right) & =\tilde{x}_{{j^{*}}^{*}}\left(t_{i}\right), \\
z_{2 \lambda}\left(t_{i}{ }^{+}\right) & =\tilde{x}_{21_{j^{*}}}\left(t_{i}\right)-L_{2_{j^{*}}} \tilde{x}_{12_{j^{*}}}\left(t_{i}\right), \\
z_{3 \lambda_{\lambda}}\left(t_{i}{ }^{+}\right) & =\tilde{x}_{22_{j^{*}}}\left(t_{i}\right)-E_{22 j^{*}} E_{2 j^{*}}^{+}\left[\begin{array}{c}
\tilde{x}_{12}\left(t_{i}\right) \\
\tilde{x}_{21}\left(t_{i}\right)
\end{array}\right] .
\end{aligned}
$$

Proof

In accordance with Theorem $2, \bar{x}_{1}$ is estimated exactly and in finite time by means of $\tilde{x}_{1_{j^{*}}}$. Thus, the following equation is stated:

$$
\nu_{j^{*}}\left(t_{i}{ }^{+}\right)=P_{1 j^{*}}\left(\tilde{x}_{1_{j^{*}}}\left(t_{i}\right)-z_{1 j^{*}}\left(t_{i}{ }^{+}\right)\right) .
$$

If reset equation (57) is applied to (24) on each switching time, then the trajectories always remain in the sliding surface and the following equality is established:

$$
\nu_{j^{*}}\left(t_{i}{ }^{+}\right)=P_{1_{j^{*}}}\left(\tilde{x}_{j_{j^{*}}}\left(t_{i}\right)-z_{1 j^{*}}\left(t_{i}{ }^{+}\right)\right)=0 .
$$

Applying the reset equation (58) to each dynamics (21) for all $\lambda \neq j^{*},(62)$ is satisfied in each switching time $t_{i}$. Thus, the state estimation for $\bar{x}_{1}$ is maintained in spite of the switchings.

From (33), to maintain the estimation for $\bar{x}_{21}(t)$ the following equality has to be satisfied:

$$
\tilde{x}_{21_{j^{*}}}\left(t_{i}\right)=z_{2_{j^{*}}}\left(t_{i}{ }^{+}\right)+L_{2_{j^{*}}} \tilde{x}_{12_{j^{*}}}\left(t_{i}\right) .
$$

Applying reset equation (59) to (33) for all $\lambda \neq j^{*}$, (63) is satisfied and $e_{21_{\lambda}}$ remains bounded as in (42).

From (43), to maintain the estimation for $\bar{x}_{22}(t)$ the following equality has to be satisfied:

$$
\tilde{x}_{22_{j^{*}}}\left(t_{i}\right)=z_{3_{j^{*}}}\left(t_{i}{ }^{+}\right)+E_{22 j^{*}} E_{2 j^{*}}^{+}\left[\begin{array}{l}
\tilde{x}_{12}\left(t_{i}\right) \\
\tilde{x}_{21}\left(t_{i}\right)
\end{array}\right] .
$$

Applying reset equation (60) to (43) for all $\lambda \neq j^{*}$, (64) is satisfied and $e_{22_{\lambda}}$ remains bounded as in (46). 


\section{FAULT DETECTION AND IDENTIFICATION}

The FDId scheme is based on the bank of observers (47)-(50) and discrete state observer (55). Once the whole state has been estimated it is possible to make a decision test on the occurrence and a possible FId ${ }^{* *}$.

\subsection{Fault Detection Scheme}

From dynamics $\bar{x}_{12}(t)$ in (16), it is obtained:

$$
\dot{\bar{x}}_{12}(t)-\left(A_{21_{j(\bar{x})}} \bar{x}_{11}(t)+A_{22_{j(\bar{x})}} \bar{x}_{12}(t)+B_{2_{j(\bar{x})}} u+E_{12_{j(\bar{x})}} \bar{f}(t)\right)=0 .
$$

Substituting $\bar{f}(t)$ and the estimated state in (65), in the fault-free case, the following expression is obtained

$$
\begin{aligned}
\left(\dot{\hat{x}}_{12}(t)+\dot{e}_{12}(t)\right)-\left[A _ { 2 1 _ { \lambda ( \hat { x } ) } } \left(\hat{x}_{11}(t)+\right.\right. & \left.e_{11}(t)\right)+A_{22_{\lambda(\hat{x})}}\left(\hat{x}_{12}(t)+e_{12}(t)\right) \\
& \left.+B_{2_{\lambda(\hat{x})}} u-E_{12_{\lambda(\hat{x})}} K_{1_{\lambda(\hat{x})}}^{*}\left(\hat{x}_{21}(t)+e_{21}(t)\right)\right]=0,
\end{aligned}
$$

where $e_{i j}=\bar{x}_{i j}-\hat{x}_{i j}, i, j=1,2$, are the estimation errors.

Let us define the following residual signal:

$$
r_{\lambda(\hat{x})}(t)=\dot{\hat{x}}_{12}(t)-\left[A_{21_{\lambda(\hat{x})}} \hat{x}_{11}(t)+A_{22_{\lambda(\hat{x})}} \hat{x}_{12}(t)+B_{2_{\lambda(\hat{x})}} u-E_{12_{\lambda(\hat{x})}} K_{1_{\lambda(\hat{x})}^{*}}^{*} \hat{x}_{21}(t)\right] .
$$

Notice that all the variables of the right-hand in (67) are available except $\dot{\hat{x}}_{12}(t)$ which can be estimated using the differentiator (24) for $n_{\mathcal{V}}=1$. In this way, it can be shown that

$$
\begin{aligned}
\left\|r(t)_{\lambda(\hat{x})}\right\| & \leq\left\|\dot{e}_{12}(t)\right\|+\left\|A_{21_{\lambda(\hat{x})}} e_{11}(t)\right\|+\left\|A_{22_{\lambda(\hat{x})}} e_{12}(t)\right\|+\left\|E_{12_{\lambda(\hat{x})}} K_{1_{\lambda(\hat{x})}^{*}}^{*} e_{21}(t)\right\|, \\
\left\|r(t)_{\lambda(\hat{x})}\right\| & \leq r_{\lambda(\hat{x})}^{+}(t), \text { a.e., }
\end{aligned}
$$

where $r_{\lambda(\hat{x})}^{+}(t)$ is a constant for each $\lambda(\hat{x})$, that depends on the sampling time $h$, the system and observer parameters. It is possible to estimate each $r_{\lambda(\hat{x})}^{+}(t)$ just calculating, by simulation, the value of the right-hand expression in (67).

Remark 8: If there exists disturbances it would be necessary to take into account the bound of the disturbances in the calculation of the threshold, i.e. $\left\|r(t)_{\lambda(\hat{x})}\right\| \leq r_{\lambda(\hat{x})}^{+}(t)+\eta_{\lambda(\hat{x})} d^{+}$, with $\eta_{\lambda(\hat{x})}$ positive known constant for each $\lambda(\hat{x})$.

Then, the decision on the occurrence of a fault $f(t)$ is carried out when the norm of $r_{\lambda(\hat{x})}(t)$ exceeds its corresponding threshold $r_{\lambda(\hat{x})}^{+}(t)$.

Remark 9: Notice that (69) is related to the smallest detectable fault and the fault detectability condition. It is clear that those faults which magnitude is less than $r_{\lambda(\hat{x})}^{+}(t)$ will not be detectable.

Thus, the time instant $t_{f}$ such that $\left\|r_{\lambda(\hat{x})}\left(t_{f}\right)\right\|>r_{\lambda(\hat{x})}^{+}(t)$ for $t_{f}>t_{j^{*}}$ with $t_{j^{*}}$ the time when the observer has converged, is defined as the fault detection time. Of course this implies that it is only possible to detect the faults after the bank of observers (47)-(50) and (55) has converged.

The following theorem establishes the class of faults that can be detected by the proposed fault detection scheme.

Theorem 8: Let the bank of observers (47)-(50) and (55), and the fault detection decision scheme (69) be applied to the system (16). For all time instant $t_{f}>t_{j^{*}}$ such that $f(t)$ satisfies

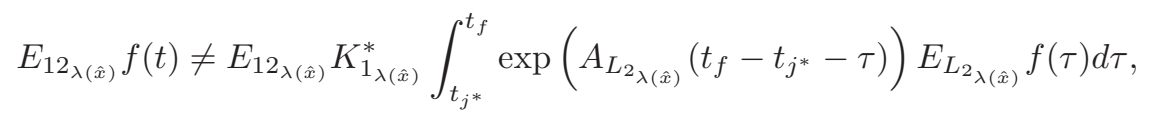

\footnotetext{
**The identification of faults implies to know the value and shape of the fault signal.
} 
then, the fault $f(t)$ will be detected, i.e. $\left\|r_{\lambda(\hat{x})}\left(t_{f}\right)\right\|>r_{\lambda(\hat{x})}^{+}(t)$, at time $t=t_{f}$.

Proof

In the faulty case, from (66) it is obtained that

$$
\begin{aligned}
\left(\dot{\hat{x}}_{12}(t)+\dot{e}_{12}(t)\right)-\left[A_{21_{\lambda(\hat{x})}}\right. & \left(\hat{x}_{11}(t)+e_{11}(t)\right)+A_{22_{\lambda(\hat{x})}}\left(\hat{x}_{12}(t)+e_{12}(t)\right) \\
& \left.+B_{2_{\lambda(\hat{x})}} u-E_{12_{\lambda(\hat{x})}} K_{1_{\lambda(\hat{x})}^{*}}^{*}\left(\hat{x}_{21}(t)+e_{21}(t)\right)\right]=E_{12_{\lambda(\hat{x})}} f(t) .
\end{aligned}
$$

Substituting the solution of (41) in (71)

$$
\begin{aligned}
\left(\dot{\hat{x}}_{12}(t)+\dot{e}_{12}(t)\right)- & {\left[A_{21_{\lambda(\hat{x})}}\left(\hat{x}_{11}(t)+e_{11}(t)\right)+A_{22_{\lambda(\hat{x})}}\left(\hat{x}_{12}(t)+e_{12}(t)\right)+B_{2_{\lambda(\hat{x})}} u\right.} \\
& -E_{12_{\lambda(\hat{x})}} K_{1_{\lambda(\hat{x})}^{*}}^{*}\left(\hat{x}_{21}(t)+e_{21}\left(t_{j^{*}}\right) \exp \left(A_{L_{2_{\lambda(\hat{x})}}} t\right)+\right. \\
& \left.\left.\int_{t_{j^{*}}}^{t_{f}} \exp \left(A_{L_{2_{\lambda(\hat{x})}}}\left(t_{f}-t_{j^{*}}-\tau\right)\right) E_{L_{2_{\lambda(\hat{x})}}} f(\tau) d \tau\right)\right]=E_{12_{\lambda(\hat{x})}} f(t) .
\end{aligned}
$$

From (72) the fault detection decision (69) is stated, i.e. $\left\|r_{\lambda(\hat{x})}(t)\right\| \leq r_{\lambda(\hat{x})}^{+}$. Notice that in the faulty case, from (72), it is obtained

$$
\begin{aligned}
& \left\|r_{\lambda(\hat{x})}(t)\right\|>\left\|\dot{e}_{12}(t)\right\|+\left\|A_{21_{\lambda(\hat{x})}} e_{11}(t)\right\|+\left\|A_{22_{\lambda(\hat{x})}} e_{12}(t)\right\| \\
& +\left\|E_{12_{\lambda(\hat{x})}} K_{1_{\lambda(\hat{x})}^{*}}^{*} e_{21}(t)\right\|+\left\|E_{12_{\lambda(\hat{x})}} f(t)\right\|, \\
& \left\|r_{\lambda(\hat{x})}(t)\right\|>r_{\lambda(\hat{x})}^{+}+\left\|E_{12_{\lambda(\hat{x})}} K_{1_{\lambda(\hat{x})}^{*}}^{*} \int_{t_{j^{*}}}^{t_{f}} \exp \left(A_{L_{\lambda_{\lambda(\hat{x})}}}\left(t_{f}-t_{j^{*}}-\tau\right)\right) E_{L_{2_{\lambda(\hat{x})}}} f(\tau) d \tau\right\| \\
& +\left\|E_{12_{\lambda(\hat{x})}} f(t)\right\|, \\
& \left\|r_{\lambda(\hat{x})}(t)\right\|>r_{\lambda(\hat{x})}^{+}(t)+\left(\gamma_{\lambda(\hat{x})}+\left\|E_{\left.12_{\lambda(\hat{x}}\right)}\right\|\right) f^{+} \text {, a.e. }
\end{aligned}
$$

Based on (72) and (75), if there exist a time instant $t_{f}>t_{j^{*}}$ such that (70) is satisfied, then it is concluded that $\left\|r_{\lambda(\hat{x})}\left(t_{f}\right)\right\|>r_{\lambda(\hat{x})}^{+}(t)$ at time $t=t_{f}$ and therefore, the fault $f(t)$ is detected.

The proposed scheme ensures the robustness of the observer w.r.t. bounded additive faults in the state estimation, maintaining the estimation error bounded by a bound of $f(t)$, leading to fault detection, in the noise-free case. In this sense, the condition (69), as it was mentioned in Remark 9, and (70) are called structural detectability conditions. Therefore, all the faults that satisfy the structural detectability conditions will be detected. In the noisy case, it is necessary to extend these conditions to get a fault detectability conditions from a sensitivity point of view.

Remark 10: Notice that (69) and (75) are not satisfied during the switching times. However, since it is possible to estimate the discrete state in the presence of faults (Theorem 6), it is possible to ignore the time instants in which these conditions are not satisfied.

\subsection{Fault Identification Scheme}

In the following, the FId problem is studied. Notice that, from (72), the following "VIE of Second Kind" could be established:

$$
\Lambda_{\lambda(\hat{x})} f(t)+\Gamma_{\lambda(\hat{x})} \int_{t_{f}}^{t} K_{\lambda(\hat{x})}(t, \tau) f(\tau) d \tau=\Phi_{\lambda(\hat{x})}(t),
$$


where

$$
\begin{aligned}
& \Lambda_{\lambda(\hat{x})}=E_{12_{\lambda(\hat{x})}}, \\
& \Gamma_{\lambda(\hat{x})}=E_{12_{\lambda(\hat{x})}} K_{1_{\lambda(\hat{x})}^{*}}^{*}, \\
& K_{\lambda(\hat{x})}(t, \tau)=\exp \left(A_{L_{2}(\hat{x})}\left(t-t_{f}-\tau\right)\right) E_{L_{2 \lambda(\hat{x})}}, \\
& \Phi_{\lambda(\hat{x})}(t)=\dot{\hat{x}}_{12}(t)-A_{21_{\lambda(\hat{x})}} \hat{x}_{11}(t)-A_{22_{\lambda(\hat{x})} \hat{x}_{12}(t)-B_{2_{\lambda(\hat{x})}} u} \\
& \quad+E_{12_{\lambda(\hat{x})}} K_{1_{\lambda(\hat{x})}^{*}} \hat{x}_{21}(t)+e_{21}\left(t_{j^{*}}\right) \exp \left(A_{L_{2 \lambda(\hat{x})}} t\right)+\epsilon(t) .
\end{aligned}
$$

The problem is to find the solution of (76), i.e. $f(t)$, taking into account that the parameters $\Lambda_{\lambda(\hat{x})}$, $\Gamma_{\lambda(\hat{x})}, K_{\lambda(\hat{x})}(t, \tau)$ and $\Phi_{\lambda(\hat{x})}(t)^{\dagger \dagger}$ are known. It is well-known that to find an analytical solution for this type of equations is difficult, sometimes impossible, and the numerical solution require an important computational effort. In this paper, the following numerical solution is given.

Consider (76) and its representation in discrete time in a time interval $\left(t_{f}, t_{f}+T\right]$, i.e.

$$
\Lambda_{\lambda(\hat{x})} f\left(t_{i}\right)+\zeta \Gamma_{\lambda(\hat{x})} \sum_{k=1}^{N} K_{\lambda(\hat{x})}\left(t_{i}, t_{k}\right) f\left(t_{k}\right)=\Phi_{\lambda(\hat{x})}\left(t_{i}\right), \forall i=1, \ldots, N
$$

where $t_{i}=t_{f}+\zeta i, \forall i=1, \ldots, N$, and $\zeta=\frac{T}{N}$. In this way, (77) can be rewritten as

$$
\mathbf{K}_{\lambda(\hat{x})}(\Lambda, \delta \Gamma, K)\left[\begin{array}{c}
f\left(t_{1}\right) \\
f\left(t_{2}\right) \\
\vdots \\
f\left(t_{N}\right)
\end{array}\right]=\left[\begin{array}{c}
\Phi_{\lambda(\hat{x})}\left(t_{1}\right) \\
\Phi_{\lambda(\hat{x})}\left(t_{2}\right) \\
\vdots \\
\Phi_{\lambda(\hat{x})}\left(t_{N}\right)
\end{array}\right]
$$

where

$$
\mathbf{K}_{\lambda(\hat{x})}(\Lambda, \delta \Gamma, K)=\left[\begin{array}{ccc}
\Lambda_{\lambda(\hat{x})}+\zeta \Gamma_{\lambda(\hat{x})} K_{\lambda(\hat{x})}\left(t_{1}, t_{1}\right) & \cdots & \zeta \Gamma_{\lambda(\hat{x})} K_{\lambda(\hat{x})}\left(t_{1}, t_{N}\right) \\
\zeta \Gamma_{\lambda(\hat{x})} K_{\lambda(\hat{x})}\left(t_{2}, t_{1}\right) & \cdots & \zeta \Gamma_{\lambda(\hat{x})} K_{\lambda(\hat{x})}\left(t_{2}, t_{N}\right) \\
\vdots & \ddots & \vdots \\
\zeta \Gamma_{\lambda(\hat{x})} K_{\lambda(\hat{x})}\left(t_{N}, t_{1}\right) & \cdots & \Lambda_{\lambda(\hat{x})}+\zeta \Gamma_{\lambda(\hat{x})} K_{\lambda(\hat{x})}\left(t_{N}, t_{N}\right)
\end{array}\right] .
$$

The FId problem is reduced to that one of solving matrix equation of the form $\mathbf{K} f=\Phi$ for $f$. However, the problem can be ill-conditioned if the condition number ${ }^{\ddagger \ddagger}$ associated with (78) is high, since, the condition number gives a bound on how inaccurate the solution $f$ will be after approximation. In particular, it is possible to think of the condition number as being (very roughly) the rate at which the solution, $f$, will change with respect to a change in $\Phi$. Thus, if the condition number is large, even a small error in $\Phi$ may cause a large error in $f$. On the other hand, if the condition number is small then the error in $f$ will not be much bigger than the error in $\Phi$.

Remark 11: The condition number is related to fault identifiability condition. It is clear that a well condition number, i.e. near 1, will allow to solve the FId problem.

In order to reduce the condition number of $\mathbf{K}$ it is possible to obtain a preconditioner $\mathbf{P}$ of matrix $\mathbf{K}$ such that $\mathbf{P}^{-1} \mathbf{K}$ has a smaller condition number than $\mathbf{K}$. The preconditioned matrix $\mathbf{P}^{-1} \mathbf{K}$ is not explicitly formed. The action of applying the preconditioner operation to a given vector need to be computed in iterative methods (see, for example [56]).

In the following simulation results are presented in order to show the workability of the proposed methods.

\footnotetext{
$\dagger_{\dagger}$ The term $\epsilon(t)=\dot{e}_{12}(t)-A_{21_{\lambda(\hat{x})}} e_{11}(t)-A_{22_{\lambda(\hat{x})}} e_{12}(t)$ contains all the computational errors produced by the state estimation process and due to sample time. Notice that, theoretically, an exact state estimation is achieved for the states $\bar{x}_{11}(t)$ and $\bar{x}_{12}(t)$.

$\ddagger \ddagger$ The condition number of a matrix measures the sensitivity of the solution of a system of linear equations to errors in the data. It gives an indication of the accuracy of the results from matrix inversion and the linear equation solution. Values of condition number near 1 indicate a well-conditioned matrix.
} 


\section{SIMULATION RESULTS}

Consider the following switched linear system to illustrate the proposed approach. The discrete state is given by

$$
j(x(t))= \begin{cases}1, & \forall x \mid H x \in[-50,2000), \\ 2, & \forall x \mid H x \in(-\infty,-50) \cup[2000, \infty),\end{cases}
$$

with $H x=-2 x_{1}(t)+5 x_{2}(t)$. The following matrices correspond to the dynamics equations (1)

$$
\begin{gathered}
A_{1}=\left[\begin{array}{cccc}
-1 & 1 & 0 & 0 \\
1 & -1 & -1 & 0 \\
1 & 1 & 1 & 0 \\
1 & 1 & 1 & -1
\end{array}\right], A_{2}=\left[\begin{array}{cccc}
-1 & -1 & 0 & 0 \\
-1 & -1 & -1 & 0 \\
1 & 2 & 1 & 0 \\
2 & 1 & 1 & -1
\end{array}\right], \\
B_{1}=\left[\begin{array}{l}
1 \\
1 \\
1 \\
1
\end{array}\right], B_{2}=\left[\begin{array}{l}
0 \\
1 \\
0 \\
1
\end{array}\right], E_{1}=\left[\begin{array}{l}
0 \\
1 \\
1 \\
1
\end{array}\right], E_{2}=\left[\begin{array}{l}
0 \\
1 \\
1 \\
0
\end{array}\right], \\
C_{1}=\left[\begin{array}{llll}
1 & 0 & 0 & 0
\end{array}\right], C_{2}=\left[\begin{array}{llll}
1.5 & 0 & 0 & 0
\end{array}\right] .
\end{gathered}
$$

The output and known input are given by $y=C_{j(x(t))} x_{1}(t)$ and $u(t)=5 \sin (t)$, respectively. The system initial conditions are set to $x(0)=\left[\begin{array}{cccc}2 & 3 & 1 & 2\end{array}\right]^{T}$ and $j(x(0))=1$. Simulations have been done in the MATLAB Simulink environment, with the Euler discretization method and sampling time $h=0.0001[\mathrm{sec}]$. It is possible to show that all assumptions stated along the paper are satisfied. The values of the designed matrices are the following

$$
\begin{gathered}
T_{1}=I_{4 \times 4}, T_{2}=\left[\begin{array}{cccc}
1.5 & 0 & 0 & 0 \\
0 & -1.5 & 0 & 0 \\
0 & 0 & 1 & 0 \\
0 & 0 & 0 & 1
\end{array}\right], K_{11}^{*}=K_{12}^{*}=\left[\begin{array}{l}
1
\end{array}\right], \bar{K}_{1}^{*}=\bar{K}_{2}^{*}=\left[\begin{array}{llll}
0 & 0 & 1 & 0
\end{array}\right], \\
L_{11}=\left[\begin{array}{cc}
33 & 267
\end{array}\right]^{T}, L_{12}=\left[\begin{array}{cc}
22 & -178
\end{array}\right]^{T}, L_{21}=-5, L_{22}=-10, \\
P_{11}=\left[\begin{array}{cc}
1 & 0 \\
-34 & 1
\end{array}\right], P_{12}=\left[\begin{array}{cc}
1.5 & 0 \\
-51 & -1.5
\end{array}\right],
\end{gathered}
$$

The HOSM differentiators in (24) are designed for $n_{\mathcal{V}}=2$ with $M_{1}$ and $M_{2}$ according to Proposition 1. The reset equations in Proposition 7 are implemented. The results for the fault-free case are depicted in Figs. 1-3.

It is possible to see that the main features of the proposed observer are clearly illustrated, i.e., finite time estimation for $x_{11}(t), x_{12}(t)$ and $j(x(t))$, and exponential estimation for $x_{21}(t)$ and $x_{22}(t)$. The decision signal $r_{\lambda(\hat{x})}^{+}(t)$ is computed by simulation and the estimating of $\dot{x}_{21}(t)$ is provided by (24) with $n_{\mathcal{V}}=1$. The behavior of decision signal $r_{\lambda(\hat{x})}^{+}(t)$ for the fault-free case is shown in Fig. 4. Notice that in the switching times condition (69) is not satisfied as it is mentioned in Remark 10.

Now, consider the following faulty scenario. The fault $f(t)=10 \sin (5.5 t)+4 \sin (3.5 t)+2 \sin (t)$ appears in $t=5[\mathrm{sec}]$. The results for the faulty case are depicted in Figs. 5-7.

It is easy to see that the finite time estimation for $x_{11}(t)$ and $x_{12}(t)$ is maintained, and the estimation error for $x_{21}(t)$ and $x_{22}(t)$ remains bounded, in spite of the switchings on the system and the fault. Notice that the fault $f(t)$ affects the behavior of the trajectories of the whole system implying changes in the discrete state; however, the continuous and discrete state estimation is maintained.

The norm of the decision signal $r_{\lambda(\hat{x})}^{+}(t)$ for different kind of faults (oscillatory, abrupt, incipient and intermittent fault) is shown in Fig. 8. The term $\left\|r_{\lambda(\hat{x})}(t)\right\|$ indicates when the fault has occurred, i.e. once the value of $\left\|r_{\lambda(\hat{x})}(t)\right\|$ exceeds the threshold $r_{\lambda(\hat{x})}^{+}(t)$, the fault is detected.

Finally, the FId scheme proposed in (78) is shown in Fig. 9 for each kind of fault. Clearly, the proposed scheme provides an approximate identification of the fault $f(t)$. Nevertheless, it is possible to improve this one reducing the condition number of the matrix $\mathbf{K}_{\lambda(\hat{x})}(\Lambda, \delta \Gamma, K)$. 

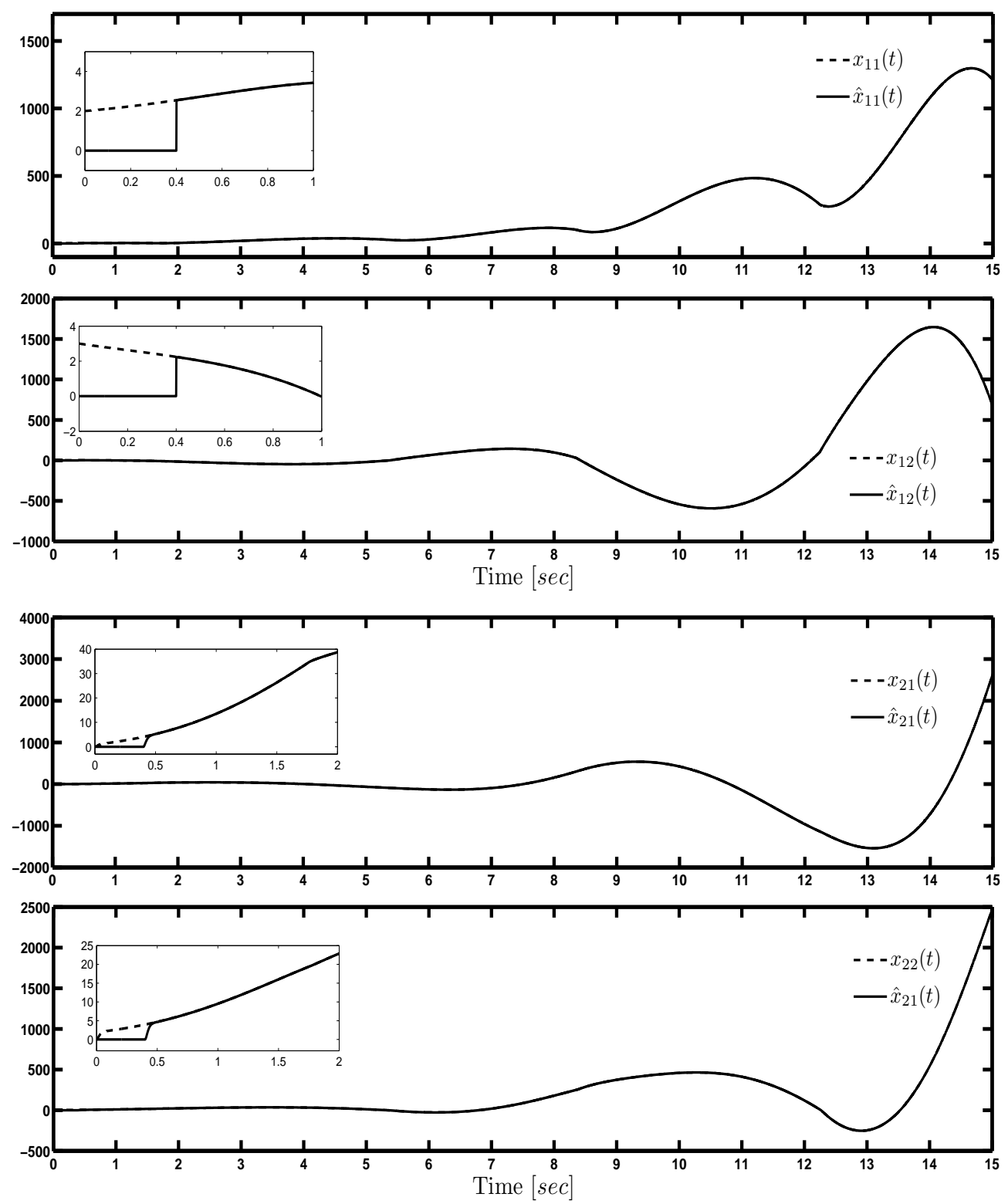

Figure 1. Continuous State Estimation. Fault-free case.

\section{CONCLUSIONS}

A solution of the problem of state estimation for NMP SS with additive faults is presented. A robust observer-based scheme for this kind of systems is proposed. The proposed state observers are based on HOSM to exactly estimate the strongly observable part, and Luenberger-like observers to estimate the remaining parts. The exact estimation of the continuous state allows us to realize a finite time and exact estimation of the discrete state in the presence of additive faults. The HOSM-based FD is composed by a residual generator accompanied by a bank of observers, and the numerical solution of a VIE allows to establish a FI scheme. Simulation results support the proposed approaches for different kind of faults. 


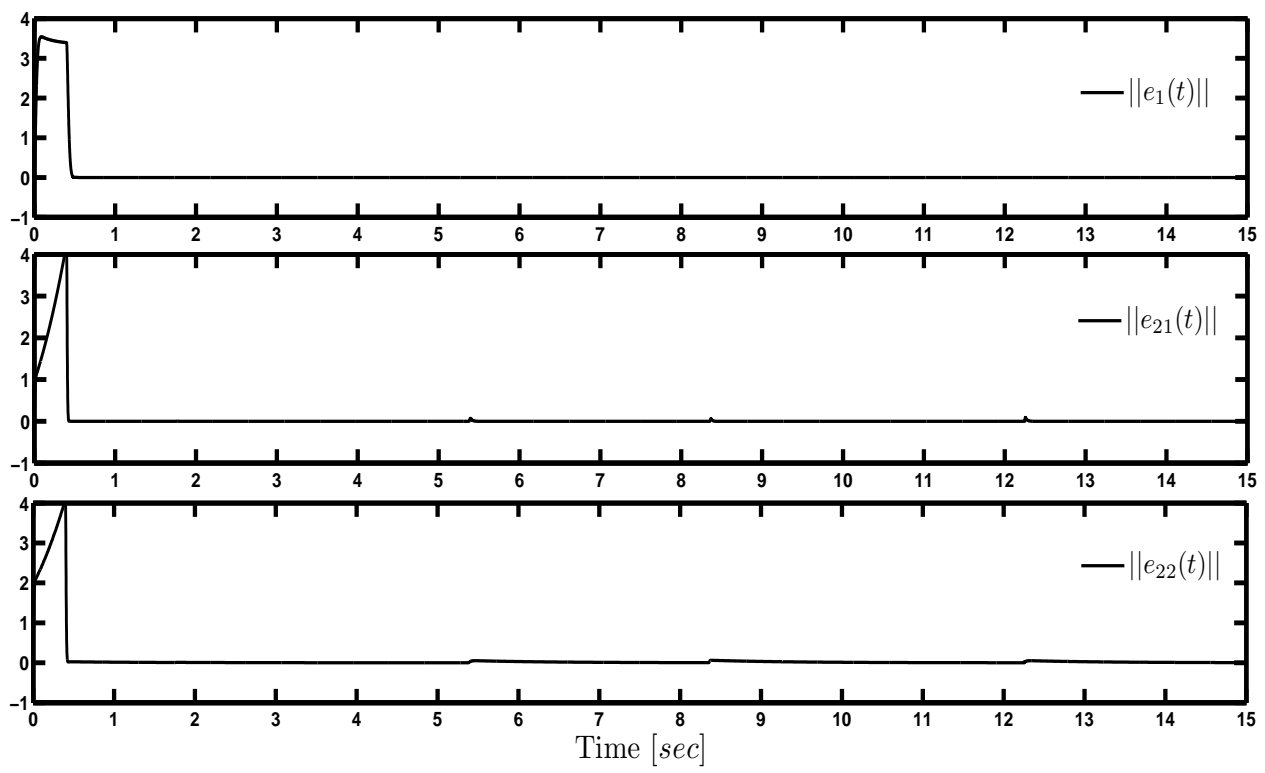

Figure 2. State Estimation Error. Fault-free case.

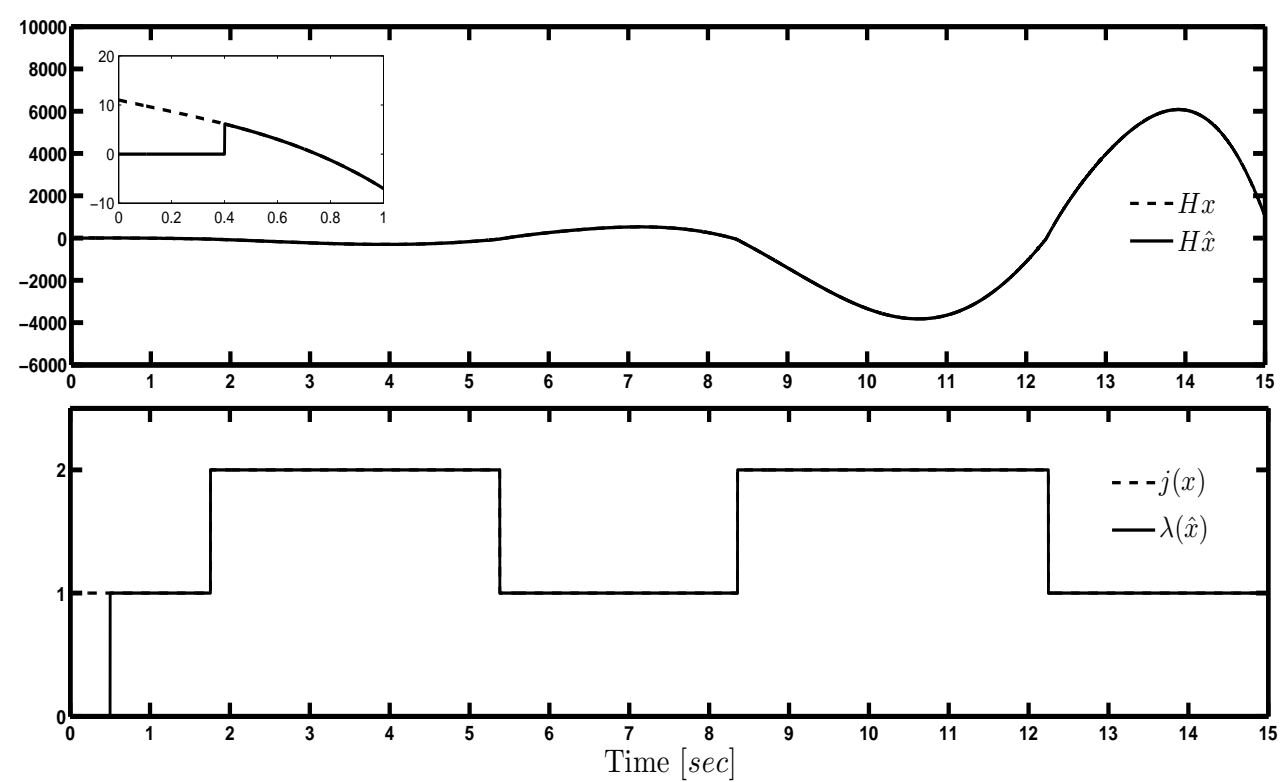

Figure 3. Switching Function and Discrete State Estimation. Fault-free case.

\section{ACKNOWLEDGEMENT}

The authors gratefully acknowledge the financial support from PAPIIT 17211, CONACyT 56819, 151855 and 270504, SIP-IPN 20110518 and CDA-IPN.

\section{REFERENCES}

1. Ríos H, Davila J, Fridman L, Efimov D. State estimation for linear switched systems with unstable invariant zeros and unknown inputs. Proceedings of the 51st IEEE Conference on Decision and Control, 2012; 5499-5504.

2. Liberzon D. Switching in Systems and Control. Systems and Control: Foundations and Applications, Birkhuser: Boston, MA, 2003 


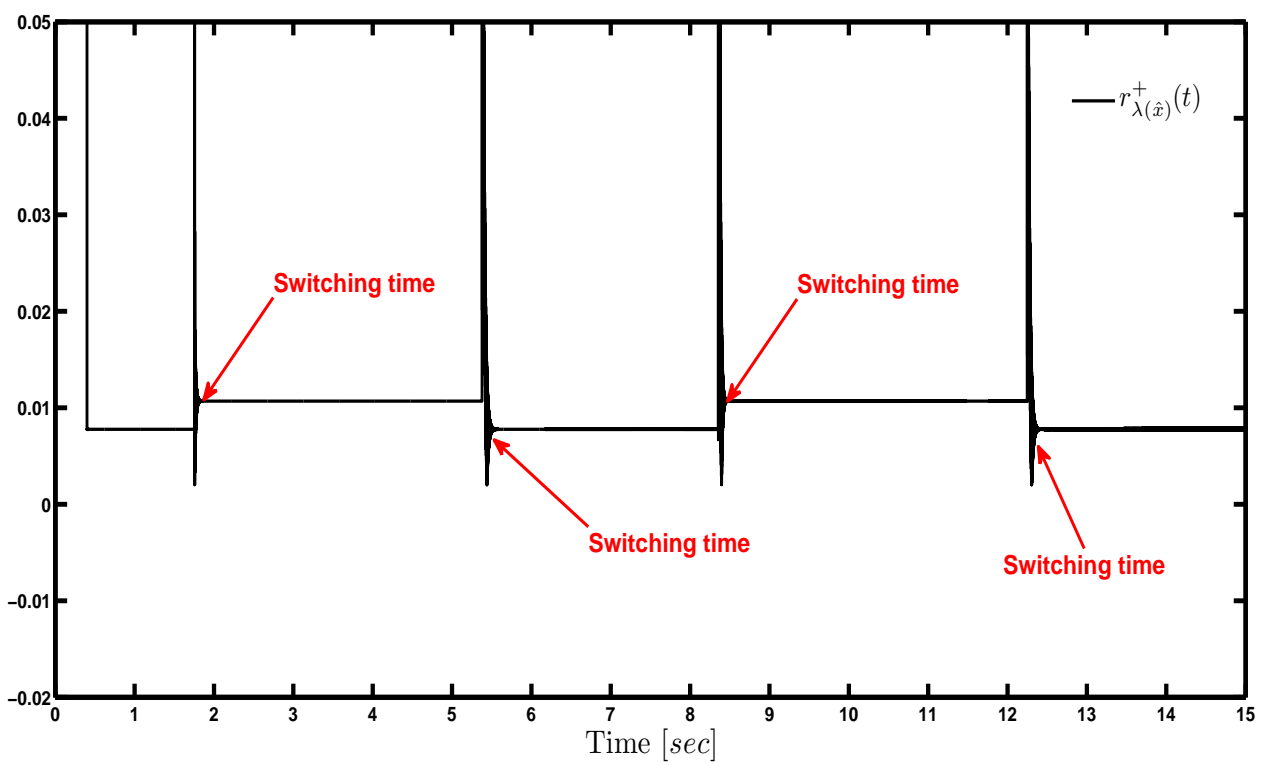

Figure 4. Fault Detection Decision. Fault-free case.

3. Lygeros J, Johansson KH, Simić SN, Zhang J, Sastry SS. Dynamical properties of hybrid automata. IEEE Transactions on Automatic Control 2003; 48(1):2-17.

4. Lin H, Antsaklis P. Stability and stabilizability of switched linear systems: A survey of recent results. IEEE Transactions on Automatic Control 2009; 54(2):308-322.

5. Alessandri A, Coletta P. Design of observers for switched discretetime linear systems. Proceedings of the 2003 American Control Conference, Denver, CO, USA, 2003; 2785-2790.

6. Pisano A, Bejarano F, Usai E. Finite-time converging jump observer for switched linear systems with unknown inputs. Nonlinear Analysis: Hybrid Systems 2011; 5(2):174-188.

7. Pina L, Botto M. Simultaneous state and input estimation of hybrid systems with unknown inputs. Automatica 2006; 42:1755-762

8. Pisano A, Bejarano F. Switched observers for switched linear systems with unknown inputs. IEEE Transactions on Automatic Control 2011; 56(3):681-686.

9. Wu L, Shi P, Gao H. State estimation and sliding-mode control of markovian jump singular systems. IEEE Transactions on Automatic Control 2010; 55(5):1213-1219.

10. Vu L, Liberzon D. Invertibility of switched linear systems. Automatica 2008; 44:949-958.

11. Defoort M, Djemai M, Floquet T, Perruquetti W. Robust finite time observer design for multicellular converters. International Journal of System Science 2011; 38(10):773-791.

12. Bobtsov A, Efimov D, Pyrkin A. Hybrid adaptive observers for locally lipschitz systems with application to mechanical oscillators. Control Applications, (CCA) \& Intelligent Control, (ISIC), 2009 IEEE, St. Petersburg, Russia, 2009; 619-624.

13. Davila J, Ríos H, Fridman L. State observation for nonlinear switched systems using nonhomogeneous high-order sliding mode observers. Asian Journal of Control July 2012; 14(4):911-923.

14. Barbot J, Saadaoui H, Djemai M, Manamanni N. Nonlinear observer for autonomous switching systems with jumps. Nonlinear Analysis: Hybrid Systems 2007; 1(4):537-547.

15. Barbot J, Djemai M, Manamanni N. State observer and observability conditions for a class of hybrid continuousdiscrete dynamic system. Proc. 46th IEEE Conf. Decision Control, 2007; 708-713.

16. Barbot J, Kang W, Xu L. On the observability of nonlinear and switched systems. Emergent Problems in Nonlinear Systems and Control, Ghosh B, Martin C, Zhou Y (eds.). Lecture Notes in Control and Information Science, Springer: Berlin Heidelberg, 2009; 199-216.

17. Pisano A, Orani N, Franceschelli M, Giua A, Usai E. Robust reconstruction of the discrete state for a class of nonlinear uncertain switched systems. Nonlinear Analysis: Hybrid Systems 2011; 5(2):220-232.

18. Floquet T, Mincarelli D, Pisano A, Usai E. Continuous and discrete state estimation in linear switched systems by sliding mode observers with residuals projection. Proceedings of the 4th IFAC Conference on Analysis and Design of Hybrid Systems, Eindhoven, The Netherlands, 2012; 265-270.

19. Ríos H, Davila J, Fridman L. High-order sliding mode observers for nonlinear autonomous switched systems with unknown inputs. Journal of the Franklin Institute 2012; 349(10):2975-3002.

20. Ding S ( (ed.)). Model-based Fault Diagnosis Techniques. Design Schemes, Algorithms, and Tools. Springer: Heidelberg, Berlin, 2008.

21. Hwang I, Kim S, Kim Y. A survey of fault detection, isolation, and reconfiguration methods. IEEE Transactions on Control Systems Technology May 2010; 18(3):636-653. 

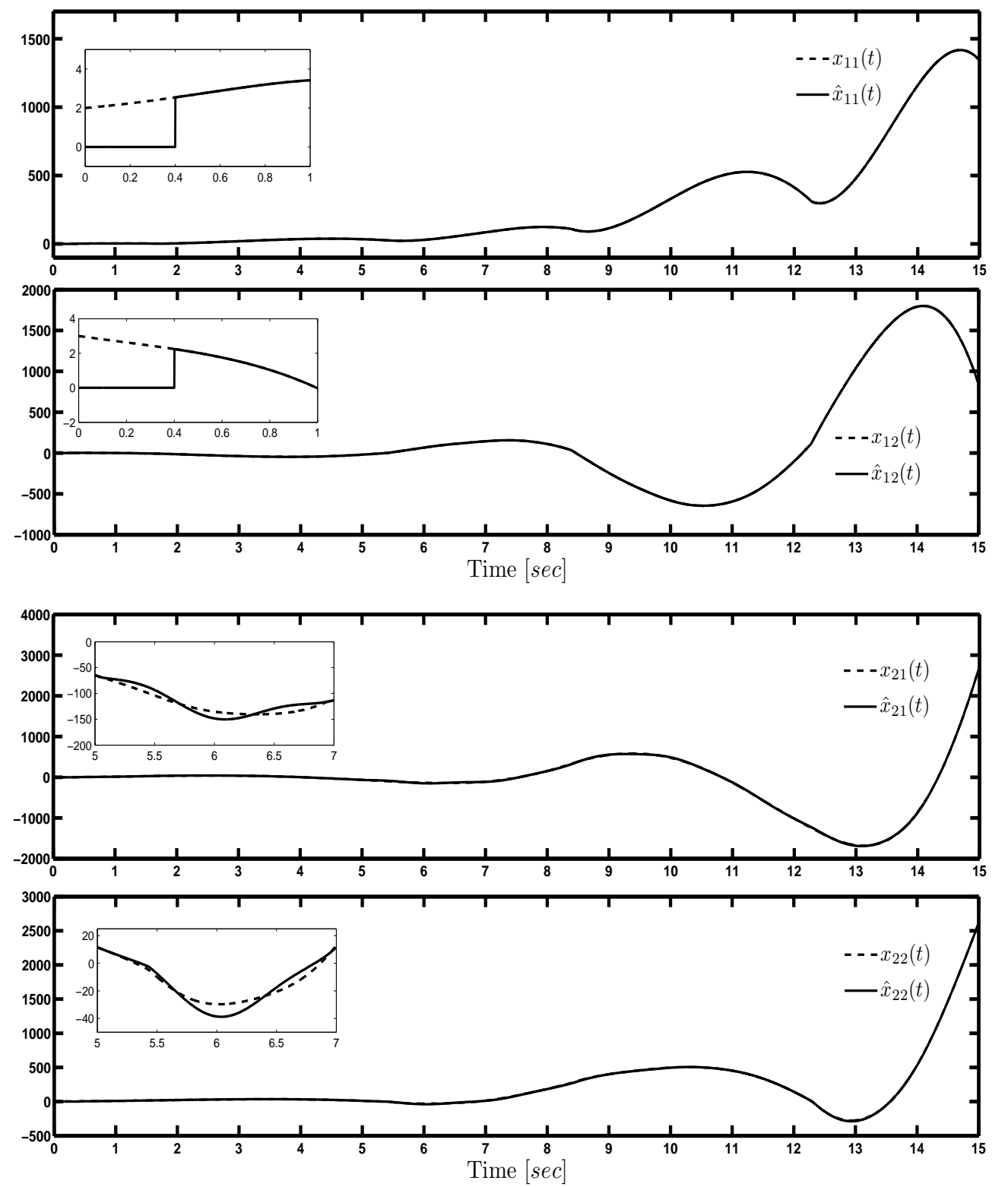

Figure 5. Continuous State Estimation. Faulty case.

22. Zolghadri A. A redundancy-based strategy for safety management in a modern civil aircraft. Control Engineering Practice 2000; 8(5):545-554.

23. Henry D, Zolghadri A. Design and analysis of robust residual generators for systems under feedback control. Automatica 2005; 41:251-264.

24. Zolghadri A, Castang F, Henry D. Design of robust fault detection filters for multivariable feedback systems. International Journal of Modeling and Simulation 2006; 26:17-26.

25. Frank P, Ding X. Survey of robust residual generation and evaluation methods in observer-based fault detection systems. International Process Control 1997; 7(6):403-424.

26. Chen J, Patton R. Robust model-based fault diagnosis for dynamic systems. Kluwer Acedmic Publishers: Boston, MA, 1999.

27. Blanke M, Zinnaert M, Lunze M, Staroswiecki M. Diagnosis and fault tolerant control. Springer: New York, 2003.

28. Zolghadri A. Advanced model-based fdir techniques for aerospace systems: Today challenges and opportunities. Progress in Aerospace Sciences 2012; 53:18-29.

29. Edwards C, Spurgeon S, Patton R. Sliding mode observers for fault detection and isolation. Automatica 2000; 36(4):541-553. 


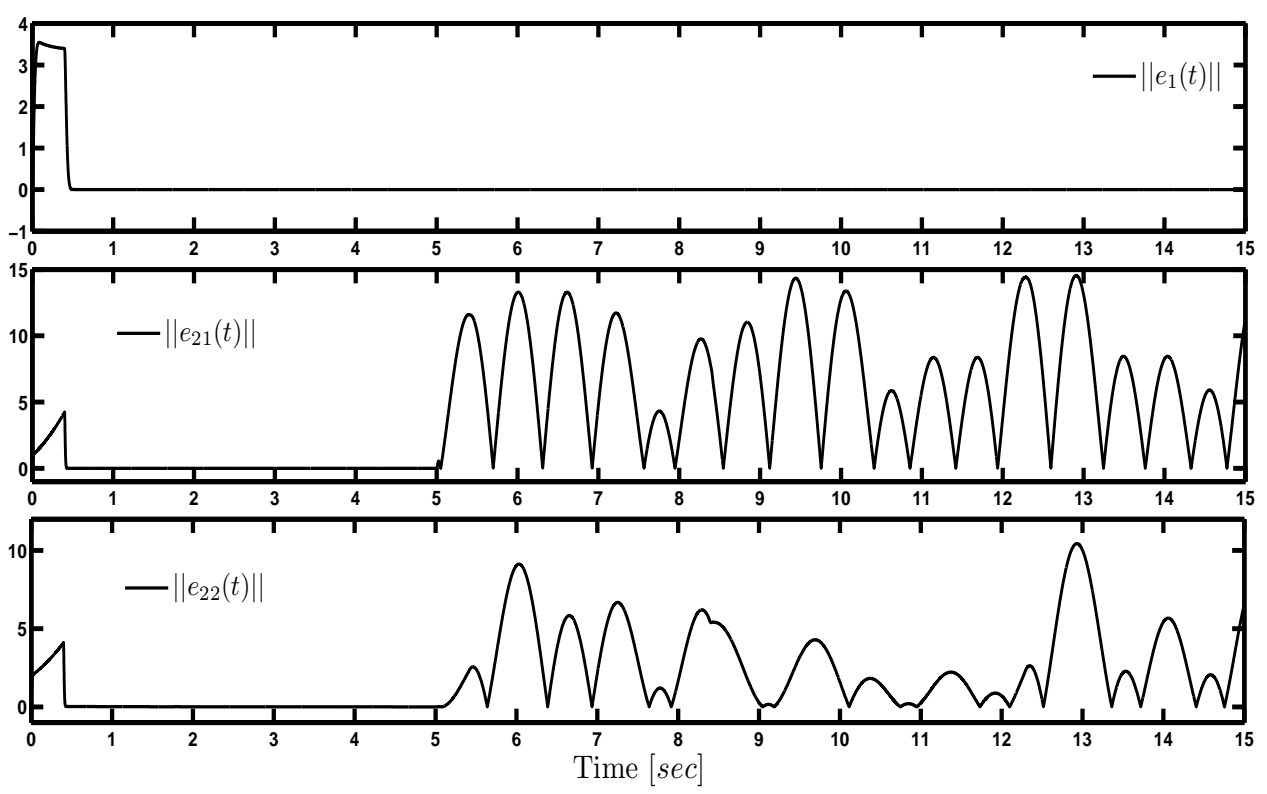

Figure 6. State Estimation Error. Faulty case.

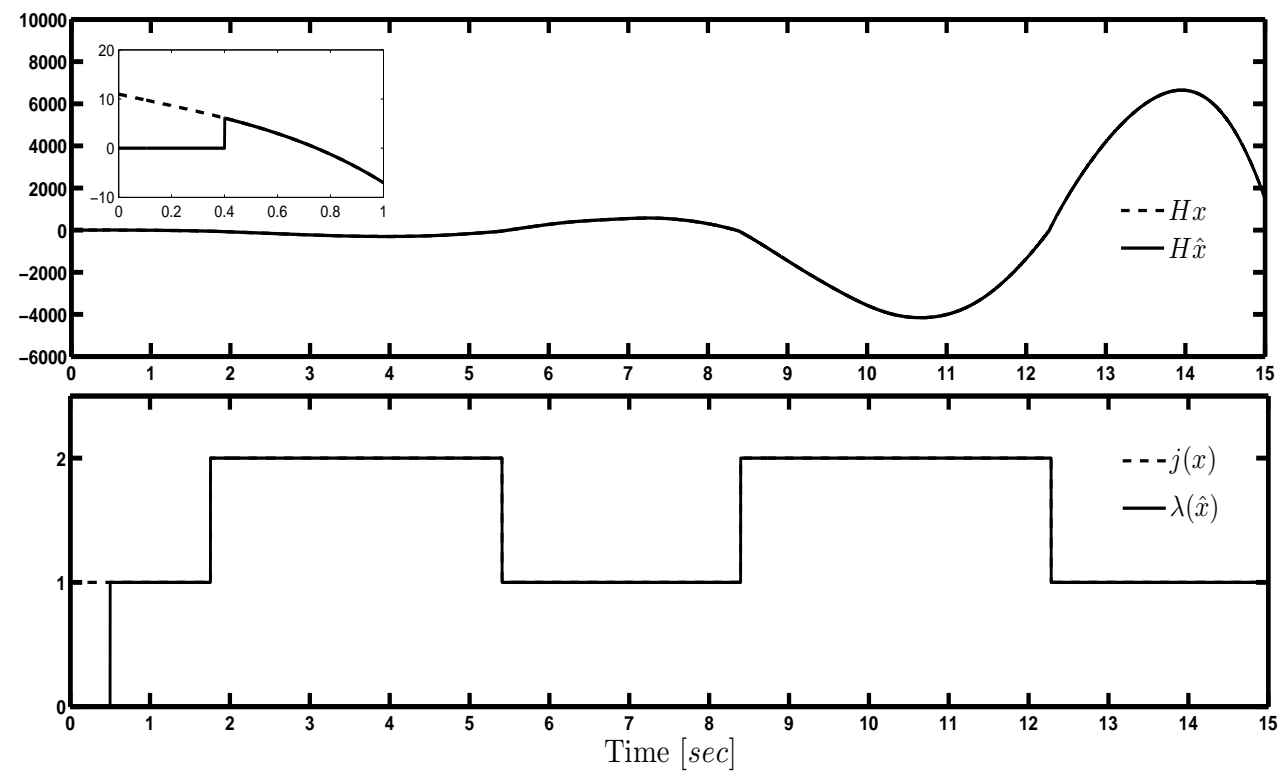

Figure 7. Switching Function and Discrete State Estimation. Faulty case.

30. Edwards C, Yan X. Nonlinear robust fault reconstruction and estimation using sliding mode observer. Automatica September 2007; 43(9):1605-1614.

31. Fridman L, Levant A, Davila J. Observation of linear systems with unknown inputs via high-order sliding-modes. International Journal of System Science 2007; 38(10):773-791.

32. Alwi H, Edwards C, Tan C. Sliding mode estimation schemes for incipient sensor faults. Automatica 2009; 45:16791865.

33. Edwards C, Alwi H, Tan C. Sliding mode methods for fault detection and fault tolerant control. 2010 Conference on Control and Fault-Tolerant Systems, 2010; 106-117.

34. Cocquempot V, El-Mezyani T, Staroswiecki M. Fault detection and isolation for hybrid systems using structured parity residuals. Proceedings of the 5th Asian Control Conference, 2004.

35. Zhao F, Koutsoukos X, Haussecker H, Reich J, Cheung P. Monitoring and fault diagnosis of hybrid systems. IEEE Transactions on Systems, Man, and Cybernetics, Part B: Cybernetics 2005; 35(6):1225-1240. 

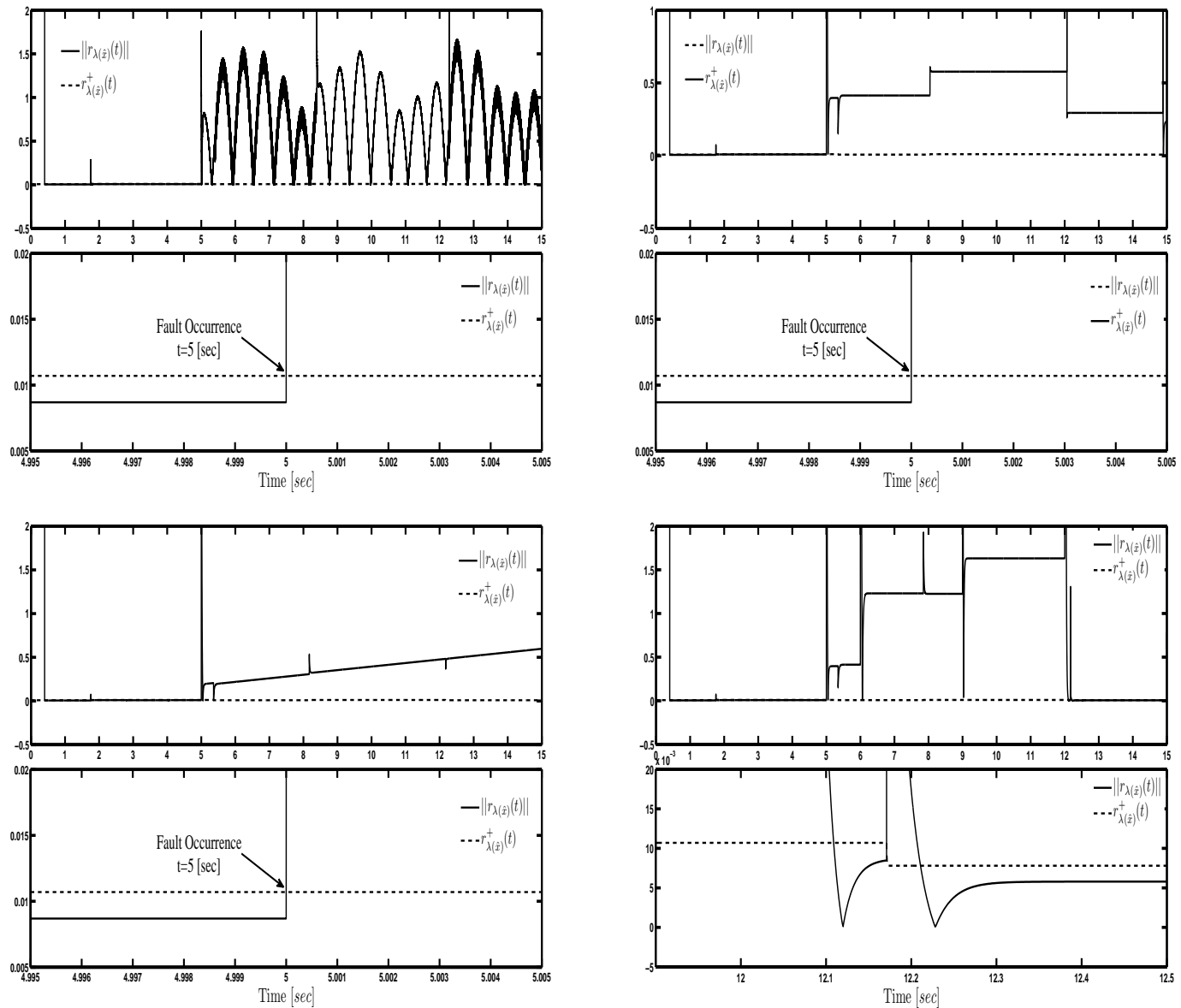

Figure 8. Fault Detection Decision. Faulty case. Notice that, in the intermittent fault, when the fault disappears, $\left\|r_{\lambda(\hat{x})}(t)\right\|$ remains less than the threshold $r_{\lambda(\hat{x})}^{+}(t)$ indicating that fault is already not present.

36. Fourlas G, Kyriakopoulos K, Krikelis N. Fault diagnosis of hybrid systems. Proc. of the 13th IEEE Mediterranean Conference on Control and Automation, 2005; 832-837.

37. Narasimhan S, Biswas G. Model-based diagnosis of hybrid systems. IEEE Transactions on Systems, Man and Cybernetics, Part A: Systems and Humans April 2007; 37(3):348-361.

38. Wang W, Li L, Zhou D, Liu K. Robust state estimation and fault diagnosis for uncertain hybrid nonlinear systems. Nonlinear Analysis: Hybrid Systems 2007; 1(1):2-15.

39. Seah C, Hwang I. Fault detection and isolation for stochastic linear hybrid systems. Proceedings of the 48th IEEE Conference on Decision and Control, 2009; 2622-2627.

40. Yang H, Cocquempot V, Jiang B. Robust fault tolerant tracking control with application to hybrid nonlinear systems. IET Control Theory \& Applications 2009; 3(2):211-224.

41. Chaib S, Boutat D, Benali A, Kratz F. Failure detection and reconstruction in switched nonlinear systems. Nonlinear Analysis: Hybrid Systems 2009; 3:225-238.

42. Belkhiat D, Messai N, Manamanni N. Robust fault detection based-observer for linear switched systems. Proceedings of the 3rd IFAC Conference on Analysis and Design of Hybrid Systems, vol. 3, Zaragoza, Spain, 2009. Doi:10.3182/20090916-3-ES-3003.00067.

43. Abdo A, Damlakhi W, Saijai J, Ding SX. Design of robust fault detection filter for hybrid switched systems. 2010 Conference on Control and Fault-Tolerant Systems, 2010; 161-166.

44. Liu W, Hwang I. Robust estimation and fault detection and isolation algorithms for stochastic linear hybrid systems with unknown fault input. IET Control Theory \& Applications 2011; 5(12):1353-1368.

45. Shtessel Y, Baev S, Edwards C, Spurgeon S. Hosm observer for a class of non-minimum phase causal nonlinear mimo systems. IEEE Transactions on Automatic Control February 2010; 55(2):543-548.

46. Hautus M. Strong detectability and observers. Linear Algebra and its Applications 1983; 50:353-368.

47. Trentelman HL, Stoorvogel AA, Hautus M. Control theory for linear systems. Springer-Verlag: London, Great Britain, 2001

48. Fridman L, Davila J, Levant A. High-order sliding-mode observation for linear systems with unknown inputs. Nonlinear Analysis: Hybrid Systems 2011; 5(2):189-205. 

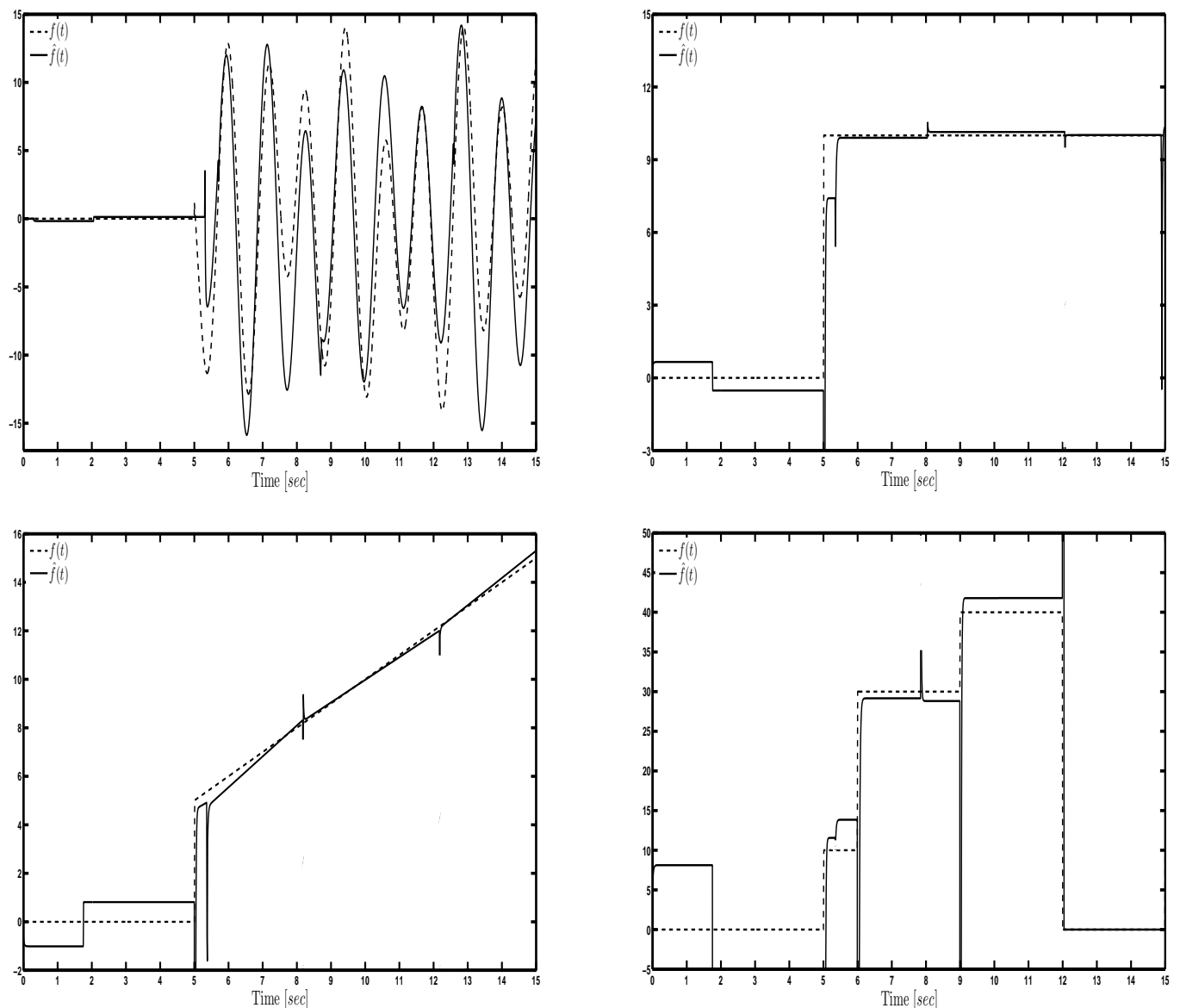

Figure 9. Fault Identification.

49. Tian Y, Floquet T, Belkoura L, Perruquetti W. Algebraic switching time identification for a class of linear hybrid systems. Nonlinear Analysis: Hybrid Systems 2011; 5(2):233-241.

50. Molinari BP. A strong controllability and observability in linear multivariable control. IEEE Transactions on Automatic Control 1976; 21(5):761-764.

51. Isidori A. Nonlinear Control Systems. Springer-Verlag: London, England, 1996

52. Bejarano F. Partial unknown input reconstruction for linear systems. Automatica 2011; 47(8):1751-1756.

53. Levant A, Livne M. Exact differentiation of signals with unbounded higher derivatives. IEEE Transactions on Automatic Control 2012; 57(4):1076-1080.

54. Levant A. High-order sliding modes: differentiation and output-feedback control. International Journal of Control 2003; 76(9-10):924-941.

55. Angulo M, Fridman L, Levant A. Robust exact finite-time output based control using high-order sliding modes. International Journal of System Science 2011; 42(11):1847-1857.

56. Boman E, Hendrickson B. Support theory for preconditioning. SIAM Journal on Matrix Analysis and Applications 2003; 25(3):694-717. 